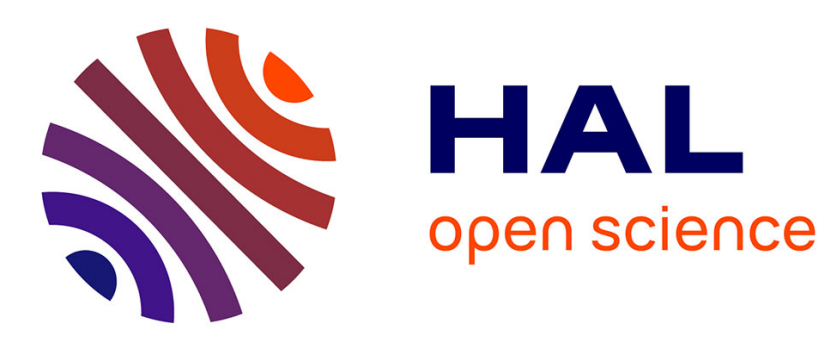

\title{
Newtonian Limit for Weakly Viscoelastic Fluid Flows
}

Didier Bresch, Christophe Prange

\section{To cite this version:}

Didier Bresch, Christophe Prange. Newtonian Limit for Weakly Viscoelastic Fluid Flows. SIAM Journal on Mathematical Analysis, 2014, 46 (2), pp.1116 - 1159. 10.1137/130923464 . hal-01915577

\section{HAL Id: hal-01915577 https://hal.science/hal-01915577}

Submitted on 2 Dec 2020

HAL is a multi-disciplinary open access archive for the deposit and dissemination of scientific research documents, whether they are published or not. The documents may come from teaching and research institutions in France or abroad, or from public or private research centers.
L'archive ouverte pluridisciplinaire HAL, est destinée au dépôt et à la diffusion de documents scientifiques de niveau recherche, publiés ou non, émanant des établissements d'enseignement et de recherche français ou étrangers, des laboratoires publics ou privés. 


\title{
NEWTONIAN LIMIT FOR WEAKLY VISCOELASTIC FLUID FLOWS
}

\author{
DIDIER BRESCH* AND CHRISTOPHE PRANGE ${ }^{\dagger}$
}

\begin{abstract}
This article addresses the low Weissenberg asymptotic analysis (Newtonian limit) of some macroscopic models of viscoelastic fluid flows in the framework of global weak solutions. We investigate the convergence of the corotational Johnson-Segalman, the FENE-P, the Giesekus and PTT models. Relying on a priori bounds coming from energy or free energy estimates, we first study the weak convergence toward the Navier-Stokes system. We then turn to the main focus of our paper, i.e. the strong convergence. The novelty of our work is to address these issues by relative entropy estimates, which require the introduction of some corrector terms. We also take into account the presence of defect measures in the initial data, uniform with respect to the Weissenberg number, and prove that they do not perturb the Newtonian limit of the corotational system.
\end{abstract}

Key words. Viscoelasticity, Weisenberg number, Deborah number, relative entropy, free energy, Newtonian limit, weak solutions

1. Introduction. This work is concerned with viscoelastic fluid flows, which have an elastic behaviour in short times, and a viscous one in large times. Such non-Newtonian fluids are ubiquitous: glaciers, Earth's mantle, dough, paint, solutions of polymers. They have a complex dynamic. For instance, phenomena such as the rod climbing effect, the tubeless siphon effect and die swell can be observed in polymeric liquids. In order to get an insight into the physics of viscoelastic fluid flows, the reader is refered to [32, 22, 30, 29].

Because of elasticity, viscoelastic fluids remember their history, which means that the dynamic of the flow at a given time depends on the past. This is in strong constrast with Newtonian fluids (i.e. purely viscous fluids). The viscoelastic relaxation time is roughly the time on which the flow remembers the past. The dimensionless number, which compares the viscoelastic relaxation time to a time scale relevant to the fluid flow, is the Weissenberg (or Deborah number) We. The bigger $W e$, the more important is the elasticity with respect to the viscosity.

The purpose of our paper is to face a problem raised by J.-C. Saut in his recent review article [34: the mathematical study of the Newtonian limit of models from non-Newtonian fluid mechanics, that is to say the limit $W e \rightarrow 0$. We focus on some macroscopic models of polymeric viscoelastic fluid flows. The works presented here are a first step toward a better understanding of the effect of a small amount of elasticity on the Newtonian dynamic of a fluid with weak regularity.

1.1. Macroscopic models of viscoelastic fluid flows. All macro-macro models we consider here are the coupling of a momentum equation on the incompressible velocity $u=u(t, x) \in \mathbb{R}^{d}$ and an equation for the symmetric stress tensor $\tau=\tau(t, x) \in M_{d}(\mathbb{R})$ (or a symmetric structure tensor $A=A(t, x) \in M_{d}(\mathbb{R})$, which has a microscopic meaning). In the sequel, we concentrate on two models: namely the corotational Johnson-Segalman model

$$
\left\{\begin{aligned}
\partial_{t} u+u \cdot \nabla u-(1-\omega) \Delta u+\nabla p & =\nabla \cdot \tau, \\
\nabla \cdot u & =0, \\
W e\left(\partial_{t} \tau+u \cdot \nabla \tau+\tau W(u)-W(u) \tau\right)+\tau & =2 \omega D(u),
\end{aligned}\right.
$$

and the FENE-P model

$$
\left\{\begin{aligned}
\partial_{t} u+u \cdot \nabla u-(1-\omega) \Delta u+\nabla p & =\nabla \cdot \tau, \\
\nabla \cdot u & =0, \\
\tau & =\frac{(b+d) \omega}{b} \frac{1}{W e}\left(\frac{A}{1-\frac{T r A}{b}}-I\right), \\
\partial_{t} A+u \cdot \nabla A-\nabla u A-A(\nabla u)^{T}+\frac{1}{W e} \frac{A}{1-\frac{T r A}{b}} & =\frac{1}{W e} I .
\end{aligned}\right.
$$

Notice that these systems are posed in $\Omega \subset \mathbb{R}^{d}$ a bounded domain, $\Omega=\mathbb{R}^{d}$ or $\Omega=\mathbb{T}^{d}$. We recall that $D(u):=\frac{\nabla u+(\nabla u)^{T}}{2}$ is the deformation tensor and that $W(u):=\frac{\nabla u-(\nabla u)^{T}}{2}$ is the vorticity

\footnotetext{
*Laboratoire de Mathématiques, Université de Savoie, 73376 Le Bourget Du Lac, France. E-mail address: didier.bresch@univ-savoie.fr

${ }^{\dagger}$ The University of Chicago, $5734 \mathrm{~S}$. University Avenue, Chicago, IL 60637, USA. E-mail address: cp@math.uchicago.edu
} 
tensor. The quantity $\partial_{t} \tau+u \cdot \nabla \tau+\tau W(u)-W(u) \tau\left(\right.$ resp. $\left.\partial_{t} A+u \cdot \nabla A-\nabla u A-A(\nabla u)^{T}\right)$ is known as the corotational (resp. upper convected) derivative of $\tau$ (resp. $A$ ).

In addition, we assume that $u$ satisfies a noslip boundary condition on $\partial \Omega$. There is no condition for $\tau$ (nor $A$ ) on the boundary. We start from the initial conditions:

$$
u(0, \cdot):=u_{0}, \quad \tau(0, \cdot):=\tau_{0}, \quad A(0, \cdot):=A_{0} .
$$

We consider only the case of Jeffrey fluids, for which $0<\omega<1$, in the framework of global in time weak solutions. The case $\omega=1$ turns out to be much more complicated (like Euler in comparison to Navier-Stokes).

Corotational model. A simple a priori energy estimate on 1.1 leads to

$$
\begin{aligned}
\omega\|u(t, \cdot)\|_{L^{2}(\Omega)}^{2}+2 \omega(1-\omega) \int_{0}^{t}\|\nabla u\|_{L^{2}(\Omega)}^{2}+\frac{W e}{2}\|\tau(t, \cdot)\|_{L^{2}(\Omega)}^{2}+\int_{0}^{t}\|\tau\|_{L^{2}(\Omega)}^{2} \\
\leq \omega\left\|u_{0}\right\|_{L^{2}(\Omega)}^{2}+\frac{W e}{2}\left\|\tau_{0}\right\|_{L^{2}(\Omega)}^{2} .
\end{aligned}
$$

This decay of energy is a consequence of the algebraic identity

$$
(\tau W(u)-W(u) \tau): \tau=0
$$

Although it is convenient from a mathematical viewpoint and greatly simplifies the analysis of the system, 1.3 points out some drawbacks of the corotational model. Indeed, as underlined in [38, this decay of energy is not relevant from a physical viewpoint. Furthermore, as noticed in [32, Chapter 3], the corotational model is unable to predict some behaviours, such as the rod climbing effect.

The existence of weak solutions to the corotational model 1.1 for $d=2$ or 3 is due to P.-L. Lions and N. Masmoudi [24]. The starting point of their analysis is the inequality (1.3). They intensively rely on the use of defect measures to pass to the limit in the product $\tau_{n} W\left(u_{n}\right)$, where $\left(u_{n}, \tau_{n}\right)$ is an approximated smooth solution to (1.1). Note that the regularity provided by 1.3 ) is barely $\tau \in L^{\infty}\left((0, \infty) ; L^{2}\right)$ and $\nabla u \in L^{2}\left((0, \infty) ; L^{2}\right)$. The key of the proof is the control of $\tau$ in $L^{\infty}\left((0, T) ; L^{q}\right)$ for a $q>2$ and $0<T<\infty$.

The initial velocity field is taken in the space $I_{p, q} \subset W^{-1, q}$ : for all $1<p, q<\infty$

$$
I_{p, q}:=\left\{u_{0} \in W^{-1, q} ;\left\|A_{q}^{-\frac{1}{2}} u_{0}\right\|_{L^{q}}+\left(\int_{0}^{\infty}\left\|A_{q} e^{-t A_{q}} A_{q}^{-\frac{1}{2}} u_{0}\right\|_{L^{q}} d t\right)^{\frac{1}{p}}<\infty\right\}
$$

where $A_{q}:=P_{q} \Delta$ is the Stokes operator with domain

$$
D\left(A_{q}\right):=\left\{u \in L^{q, \sigma} ; \nabla^{2} u \in L^{q},\left.u\right|_{\partial \Omega}=0\right\}
$$

$P_{q}$ being the Helmholtz projector on $L^{q, \sigma}$ with domain $L^{q}$. The space $L^{q, \sigma}$ is the closure of the space $\left\{v \in C_{0}^{\infty}(\Omega): \nabla \cdot v=0\right\}$ in $L^{q}$. For more properties about the space $I_{p, q}$ and the Stokes operator, we refer to [14.

In more details, their existence result reads:

Result A (P.-L. Lions, N. Masmoudi). There exists a global weak solution $(u, \tau)$ of 1.1 satisfying the energy inequality (1.3) such that for all $0<T<\infty$,

$$
\nabla u \in L^{p}\left((0, T) ; L^{q}\right) \quad \text { and } \quad \tau \in C^{0}\left([0, \infty) ; L^{q}\right),
$$

provided that $\tau_{0} \in L^{q}$ and $u_{0} \in I_{p, q}$,

- for some $2<q<+\infty, 1<p<+\infty$, if $d=2$,

- and for some $2<q \leq 3,1<p \leq \frac{q}{2 q-3}$, if $d=3$. 
FENE-P model. This model is one of the many closure approximations of the microscopic FENE (Finite Extensible Nonlinear Elastic) dumbbel model (see [11, 12]). Its low computational costs, compared to micro-macro models, and its acceptable predictions make it a widely used model for numerical simulation of viscoelastic fluid flows. However, as pointed out in [19, it does not capture all the physics of the microscopic model. Note that the parameter $b$ in 1.2 relates to the extensibility of the elastic dumbbels at the microscopic scale.

The energy is replaced by a non-trivial free energy (or entropy), which has been known from physicists since the work of L. E. Wedgewood and R. B. Bird [39] (see also [38, 30]). D. Hu and T. Lelièvre in [17] have recently rediscovered this entropy and showed that it decays in time:

$$
\begin{aligned}
& \frac{1}{2}\|u(t, \cdot)\|_{L^{2}(\Omega)}^{2}+(1-\omega) \int_{0}^{t}\|\nabla u\|_{L^{2}(\Omega)}^{2} \\
& \quad+\frac{\omega(b+d)}{2 b} \frac{1}{W e} \int_{\Omega}\left[-\ln (\operatorname{det} A)-b \ln \left(1-\frac{\operatorname{Tr}(A)}{b}\right)+(b+d) \ln \left(\frac{b}{b+d}\right)\right](t) \\
& \quad+\frac{\omega(b+d)}{2 b} \frac{1}{W e^{2}} \int_{0}^{t} \int_{\Omega}\left[\frac{\operatorname{Tr} A}{\left(1-\frac{\operatorname{Tr} A}{b}\right)^{2}}-\frac{2 d}{\left.1-\frac{\operatorname{TrA}}{b}+\operatorname{Tr}\left(A^{-1}\right)\right]}\right. \\
& \leq \frac{1}{2}\left\|u_{0}\right\|_{L^{2}(\Omega)}^{2}+\frac{\omega(b+d)}{2 b} \frac{1}{W e} \int_{\Omega}\left[-\ln \left(\operatorname{det} A_{0}\right)-b \ln \left(1-\frac{\operatorname{Tr}\left(A_{0}\right)}{b}\right)+(b+d) \ln \left(\frac{b}{b+d}\right)\right]
\end{aligned}
$$

Based on this decay, N. Masmoudi [26, has achieved an existence result for the system (1.2). The fundamental point is that the decay of the entropy 1.5$)$ yields a control of the $L^{2}((0, \infty) \times \Omega)$ norm of $\tau$.

Result B (N. Masmoudi). Let $d \geq 2$. Assume that $u_{0} \in L^{2}$ is a divergence free vector field and that $A_{0}=A_{0}(x)$ is a symmetric positive definite matrix with $\operatorname{Tr} A_{0}<b$ and such that

$$
\int_{\Omega}\left[-\ln \left(\operatorname{det} A_{0}\right)-b \ln \left(1-\frac{\operatorname{Tr} A_{0}}{b}\right)+(b+d) \ln \left(\frac{b}{b+d}\right)\right]<\infty .
$$

Then, there exists a global weak solution $(u, A, \tau)$ to 1.2 satisfying $(1.5)$, such that

$$
u \in L^{\infty}\left((0, \infty) ; L^{2}\right) \cap L^{\infty}\left((0, \infty) ; \dot{H}^{1}\right), A \in L^{\infty}((0, \infty) \times \Omega) \text { and } \tau \in L^{2}((0, \infty) \times \Omega) .
$$

1.2. Outline of our results. The existence theorems of global weak solutions open the way to the asymptotic analysis at low Weissenberg number. From a formal perspective, it is easy to see that the velocity field $u$ of the non-Newtonian fluid model 1.1 converges toward a solution $u^{0}$ of the Navier-Stokes system

$$
\left\{\begin{array}{rll}
\partial_{t} u^{0}+u^{0} \cdot \nabla u^{0}-\Delta u^{0}+\nabla p^{0} & =0, & \Omega \\
\nabla \cdot u^{0} & =0, & \Omega \\
u^{0} & =0, & \partial \Omega .
\end{array}\right.
$$

Notice that the noslip condition is compatible with the limit, so that no boundary layers are involved in this limit (at least at the leading order in $W e$ ). That is why, we state our results for the whole space domain $\Omega=\mathbb{R}^{d}$.

As far as we know, the Newtonian limit of non-Newtonian fluids has only been studied in the context of strong solutions. First results in the direction of a better understanding of this limit have been reached by J.-C. Saut in 33 for Maxwell type flows (no diffusion term in the momentum equation) in the linear regime. The only other result we are aware of is the one of L. Molinet and R. Talhouk [27] for strong solutions of Johnson-Segalman systems (including the corotational and the Oldroyd-B systems). For these models no energy of the type of (1.3) is available in general, so they rely on a splitting in low and high frequencies at a cut-off frequency depending on $W e$.

The originality of our work is to address the Newtonian limit in the framework of weak solutions relying only on energy (or free energy) methods. Thus our results do not ask for more smoothness than the natural regularity available. 
The first logical step in our study of the limit is to investigate the weak convergence. For the corotational and the FENE-P models, we easily obtain the weak convergence toward the NavierStokes system. More intricate calculations of relative entropies involving higher-order corrector terms make it then possible to achieve strong convergence results. The latter are the main focus of our paper.

1.2.1. Newtonian limit: weak convergence. The mathematical justification of the formal asymptotics requires a priori bounds uniform in $W e$. Some bounds, like the $L^{\infty}\left((0, \infty) ; L^{2}\right)$ bound on $\tau$ for (1.1), are not uniform in $W e$. They were usefull for the Cauchy theory, but are useless for the Newtonian limit.

Notice that the initial data $u_{0}, A_{0}$ and $\tau_{0}$ may depend on $W e$. In order to get uniform bounds in $W e$, we have to assume that initial data is well-prepared, in a sense to be made precise later on. We always start from data meeting the conditions of Result $\mathrm{A}$ or $\mathrm{B}$ leading to the existence of weak solutions. We state the weak convergence results for a bounded domain $\Omega$; the results being the same for the whole space or periodic boundary conditions.

Our first result is concerned with the weak convergence in the corotational system.

Proposition 1.1. Let $d=2$, 3. Let $(u, \tau)$ be a weak solution of $(1.1$ in the sense of Result A. Assume that

$$
\left\|u_{0}\right\|_{L^{2}(\Omega)}^{2}+\frac{W e}{2}\left\|\tau_{0}\right\|_{L^{2}(\Omega)}^{2}=O(1)
$$

Then, there exist

$$
u^{0} \in L^{\infty}\left((0, \infty) ; L^{2, \sigma}\right) \cap L^{2}\left((0, \infty) ; \dot{H}^{1}\right) \quad \text { and } \quad \tau^{0} \in L^{2}\left((0, \infty) ; L^{2}\right)
$$

such that $u$ (resp. $\tau$ ) converges to $u^{0}$ (resp. $\tau^{0}$ ) at least in the sense of distribution, where $u^{0}$ is a weak solution of the Navier-Stokes system (1.6) and $\tau^{0}=2 \omega D\left(u^{0}\right)$.

We turn to the weak convergence for the FENE-P model. It is formally clear that $A$ converges to $A^{0}:=\frac{b}{b+d} I$. The key to the convergence of $u$ is a bound on $\tau$ in $L^{2}((0, \infty) \times \Omega)$ uniform in $W e$, deduced from the decay of the free energy (1.5).

Proposition 1.2. Let $d=2$, 3. We consider a global weak solution $(u, A, \tau)$ of 1.2 in the sense of Result $B$. Assume that $\left\|u_{0}\right\|_{L^{2}(\Omega)}=O(1)$. Then we get several convergence results in the limit $W e \rightarrow 0$.

- Assume that initial data is ill-prepared in the sense that

$$
\int_{\Omega}\left[-\ln \left(\operatorname{det} A_{0}\right)-b \ln \left(1-\frac{\operatorname{Tr} A_{0}}{b}\right)+(b+d) \ln \left(\frac{b}{b+d}\right)\right]=O(1) .
$$

Then $A$ tends to $A^{0}$ in $L^{2}\left((0, \infty) ; L^{2}(\Omega)\right)$ and

$$
\left\|A-A^{0}\right\|_{L^{2}\left((0, \infty) ; L^{2}(\Omega)\right)}=O(\sqrt{W e}) .
$$

- Assume furthermore that initial data is well-prepared namely

$$
\int_{\Omega}\left[-\ln \left(\operatorname{det} A_{0}\right)-b \ln \left(1-\frac{\operatorname{Tr} A_{0}}{b}\right)+(b+d) \ln \left(\frac{b}{b+d}\right)\right]=O(W e) .
$$

Then we have the following improved convergences:

$$
\begin{gathered}
\left\|A-A^{0}\right\|_{L^{\infty}\left((0, \infty) ; L^{2}(\Omega)\right)}=O(\sqrt{W e}), \\
\left\|A-A^{0}\right\|_{L^{2}\left((0, \infty) ; L^{2}(\Omega)\right)}=O(W e) .
\end{gathered}
$$

Moreover, $\tau$ is bounded uniformly in $L^{2}((0, \infty) \times \Omega)$, and $u$ (resp. $\tau$ ) converges in the sense of distributions toward $u^{0}$ (resp. $2 \omega D\left(u^{0}\right)$ ), where $u^{0}$ satisfies the Navier-Stokes system (1.6).

Before coming to the strong convergence, let us state a slight generalization of Proposition 1.1 allowing to handle the case of oscillating initial data $\left(u_{0, n}, \tau_{0, n}\right)$. For the sake of easiness, we temporarily consider initial data independent of $W e$ and treat only the case $d=2$. We assume that 
$u_{0, n}$ strongly converges in $L^{2}(\Omega)$ toward $u_{0}$, and that $\tau_{0, n}$ is uniformly equiintegrable in $L^{2}(\Omega)$. In particular, we do not assume that $\tau_{0, n}$ converges strongly in $L^{2}(\Omega)$. We then call $\left(u_{n}, \tau_{n}\right)$ the associated weak solution of (1.1), which satisfies the energy inequality (1.3). We show that passing to the limit on $n$ introduces defect measures in the limit system. These defect measures are due to the oscillations of the initial data $\tau_{0, n}$. We prove that they disappear in the limit $W e \rightarrow 0$.

Proposition 1.1 BIS. Let $d=2$ and $\Omega=\mathbb{R}^{2}$. The result is in two points:

- Limit $n \rightarrow \infty$. There exists

$$
u \in L^{\infty}\left((0, \infty) ; L^{2, \sigma}\right) \cap L^{2}\left((0, \infty) ; \dot{H}^{1}\right), \quad \text { and } \quad \tau \in L^{\infty}\left((0, \infty) ; L^{2}\right),
$$

such that $\left(u_{n}, \tau_{n}\right)$ tends to $(u, \tau)$ at least in the sense of distributions and $(u, \tau)$ satisfies the system

$$
\left\{\begin{aligned}
\partial_{t} u+u \cdot \nabla u-(1-\omega) \Delta u+\nabla p & =\nabla \cdot \tau \\
\nabla \cdot u & =0 \\
W e\left[\partial_{t} \tau+u \cdot \nabla \tau+\tau W(u)-W(u) \tau+\left(\begin{array}{cc}
-\varepsilon & \frac{1}{2} \delta \\
-\frac{1}{2} \delta & \varepsilon
\end{array}\right)\right]+\tau & =2 \omega D(u)
\end{aligned}\right.
$$

with defect measures $\delta, \varepsilon \in L_{l o c}^{1}\left((0, \infty) ; L^{1}\right)$.

- Limit $W e \rightarrow 0$. There exists

$$
u^{0} \in L^{\infty}\left((0, \infty) ; L^{2, \sigma}\right) \cap L^{2}\left((0, \infty) ; \dot{H}^{1}\right) \quad \text { and } \quad \tau^{0} \in L^{2}\left((0, \infty) ; L^{2}\right),
$$

solving the Navier-Stokes system (1.6) in the sense of distributions and such that $(u, \tau)$ converges weakly toward $\left(u^{0}, \tau^{0}\right)$. This result is quite distant from the main focus of our paper. It is a natural continuation of some techniques involved in the article 24]. We therefore postpone its proof to the Appendix A. The main difficulty is to get uniform in $W e$ a priori estimates on the defect measures $\delta$ and $\varepsilon$, so as to pass to the limit in (1.11).

1.2.2. Newtonian limit: strong convergence. Our strong convergence results always follow from the same two steps: first we build an ansatz for $u, A$ and $\tau$, and then we compare this approximation to $u, A$ and $\tau$ in an appropriate norm derived from the energy, or the free energy associated to the system. This is the leitmotiv of relative entropy (or modulated energy) methods. Since the pioneering works of C. Dafermos [7, 8, of R. Di Perna [10] and of H.-T. Yau [40, relative entropy methods have become a crucial and widely used tool in the study of asymptotic limits to kinetic models in the contex of hydrodynamics [25, 15, 2, of the quasineutral limit for the VlasovPoisson system [3, 16, of the behavior of shocks for systems of conservation laws [23] and of the stability in thermomechanical theories [20, 9. They have also been successfully implemented in the approximation of incompressible fluids by hyperbolic systems [4, 28]; see also [37, 21] for an expository of the general method, which is close to ours, and the use of corrector terms. Let us also mention the use of relative entropies for the study of the long-time behavior of some micro-macro models for dilute solutions of polymers, and the convergence to equilibrium by B. Jourdain, C. Le Bris, T. Lelièvre and F. Otto [18]. Relative entropy methods are also the key to the estimates of F. Otto and A. Tzavaras in 31 .

The very rough idea is work with an energy (resp. free energy) $\mathfrak{e}=\mathfrak{e}(u, A, \tau)$ like the one in the left hand side of 1.3 (resp. (1.5p). Notice that $\mathfrak{e}(u, A, \tau)=\mathfrak{e}_{1}(u)+\mathfrak{e}_{2}(A)+\mathfrak{e}_{3}(\tau)$. The decisive point, is that the functions $\mathfrak{e}_{i}$, for $i=1, \ldots 3$ are globally convex. Thus, one can make a Taylor expansion of $\mathfrak{e}$ around say $(\tilde{u}, \tilde{A}, \tilde{\tau})$, and get that the quantity

$$
\mathfrak{E}(u, A, \tau):=\mathfrak{e}(u, A, \tau)-\mathfrak{e}(\tilde{u}, \tilde{A}, \tilde{\tau})-\nabla \mathfrak{e}_{1}(\tilde{u}) \cdot(u-\tilde{u})-\nabla \mathfrak{e}_{2}(\tilde{A}) \cdot(A-\tilde{A})-\nabla \mathfrak{e}_{3}(\tilde{\tau}) \cdot(\tau-\tilde{\tau})
$$

is positive and controls the norm of

$$
|u-\tilde{u}|^{2}+\alpha|A-\tilde{A}|^{2}+\beta|\tau-\tilde{\tau}|^{2},
$$

with $\alpha, \beta \geq 0, \alpha=0$ (resp. $\beta=0$ ) for the corotational (resp. for the FENE-P) model. The quantity $\mathfrak{E}$ is called the relative entropy (or modulated energy). Its control is based on an explicit computation of its total time derivative in order to establish a Gronwall type inequality. These 
computations may be quite tricky (especially in the case of the FENE-P system), as they involve the algebraic structure of the equations 1.1 or 1.2 .

This procedure yields an error estimate between $u$ (resp. $A, \tau)$ and its approximation. The convergence result holds for solutions of $(1.1)$ or $(1.2)$ with very low regularity, typically weak solutions. However, in order to carry out the estimates of the relative entropies, we need quite a lot regularity on the profiles of our ansatz.

The first of our two theorems below handles the case of the corotational system.

THEOREM 1.3. Let $d=2,3, u_{0} \in H^{4, \sigma}(\Omega)$ independent of $W e$ and $\tau_{0} \in L^{2}(\Omega) \cap L^{q}(\Omega)$, with $2<q \leq 3$. Notice that $\tau_{0}$ may depend on We in the following sense: $\left\|\tau_{0}\right\|_{L^{2}(\Omega)}=O(1)$. Let also

$$
u \in L^{\infty}\left((0, \infty) ; L^{2, \sigma}\right) \cap L^{2}\left((0, \infty) ; \dot{H}^{1}\right), \quad \text { and } \quad \tau \in L^{\infty}\left((0, \infty) ; L^{2}\right) \cap L_{l o c}^{\infty}\left((0, \infty) ; L^{q}\right)
$$

be global weak solutions to (1.1) in the sense of Result $A$ associated to the initial data $u_{0}$ and $\tau_{0}$. Then, there exists $0<T^{*}<\infty$ independent of $W$ e and

$$
u^{0} \in L^{\infty}\left((0, \infty) ; L^{2, \sigma}\right) \cap L^{2}\left((0, \infty) ; \dot{H}^{1}\right)
$$

a global weak solution of $(1.6)$ associated to the initial data $u_{0}$, such that, in addition, $u^{0}$ belongs to $L^{\infty}\left((0, T) ; H^{4}\right)$ for all $0<T<T^{*}$. Moreover, for all $0<T<T^{*}$,

$$
\begin{aligned}
\sup _{0<t<T}\left(\omega \| u(t, \cdot)-u^{0}(t, \cdot)\right. & \left\|_{L^{2}}^{2}+\omega(1-\omega) \int_{0}^{t}\right\| \nabla\left(u-u^{0}\right) \|_{L^{2}}^{2} \\
& \left.+\frac{W e}{2}\left\|\tau(t, \cdot)-\tau^{0}(t, \cdot)\right\|_{L^{2}}^{2}+\frac{1}{2} \int_{0}^{t}\left\|\tau-\tau^{0}\right\|_{L^{2}}^{2}\right)^{\frac{1}{2}}=O(\sqrt{W e}) .
\end{aligned}
$$

Let us comment on this theorem:

- The proof is done in the case when $\Omega=\mathbb{R}^{3}$. It is not hard to adapt our arguments to the easier case $\Omega=\mathbb{R}^{2}$. Furthermore, as no boundary condition is prescribed on $\tau$, and as $u^{0}=0$ can be imposed on the boundary for the limit velocity field, there is no boundary layer in the limit $W e \rightarrow 0$. Thus, our analysis extends straightforwardly to the case when $\Omega$ is a bounded domain.

- We assume that $u_{0}$ does not depend on $W e$ only in order to alleviate the proof. Of course, one can start from an initial data for $u$ depending on $W e$ and get the convergence 1.12 on condition that one assumes

$$
\left\|u(0, \cdot)-u^{0}(0, \cdot)\right\|_{L^{2}(\Omega)}=O(\sqrt{W e}) .
$$

- Our result does not require further regularity for $(u, \tau)$ than the natural regularity yielded by (1.3). However, it is quite demanding on the limit profile $u^{0}$. It might be possible to weaken the regularity requirements on the initial velocity field. In particular, we do not take advantage of the regularizing effect of the Navier-Stokes equation, which yields $u^{0} \in L^{2}\left((0, T) ; H^{5}\right)$, because we need the $L^{\infty}$ bound in time.

- The regularity needed on $u^{0}$ in order to carry out the computations of the proof is the reason why the convergence result only holds as long as $u^{0}$ remains sufficiently regular.

- Note that nothing is prescribed on the initial stress tensor $\tau_{0}$, except the requirements to get a global weak solution to (1.1). In other words, Theorem 1.3 is a convergence result for ill-prepared data $\tau_{0}$.

- Our theorem complements the study of L. Molinet and R. Talhouk [27]. They manage to get a convergence result of strong solutions for ill-prepared data, in the case when the equation on $\tau$ has the additional term $a(D(u) \tau+\tau D(u))$, with $-1 \leq a \leq 1$. Our system (1.1) corresponds to $a=0$. In the general case, $a \neq 0$, there is no known energy associated to the system. Hence their proof, unlike ours, relies on a cutting up of $u$ and $\tau$ in low and high frequencies.

- This convergence result can be used, in the two-dimensional case, to prove the existence of global in time strong solutions to (1.1) for We small enough. Of course, we would rely on the global existence of strong solutions to the Navier-Stokes system when $d=2$. 
The proof of Theorem 1.3 serves as a guideline for the main result of our paper, which is concerned with the strong convergence of the FENE-P system:

THEOREM 1.4. Let $d=2$, 3. Assume that $u_{0} \in H^{4, \sigma}(\Omega)$ is independent of We, that $A_{0} \in$ $L^{2}(\Omega)$ is symmetric positive definite and $\operatorname{Tr} A_{0}<b$. Assume furthermore that the initial data is well-prepared in the sense that

$$
\begin{aligned}
& \frac{\omega(b+d)}{2 b} \frac{1}{W e} \int_{\Omega}\left[-\ln \left(\operatorname{det} A_{0}\right)-b \ln \left(1-\frac{\operatorname{Tr} A_{0}}{b}\right)+(b+d) \ln \left(\frac{b}{b+d}\right)\right] \\
&=O(W e) .
\end{aligned}
$$

Let

$$
u \in L^{\infty}\left((0, \infty) ; L^{2}\right) \cap L^{2}\left((0, \infty) ; \dot{H}^{1}\right), \quad A \in L^{\infty}((0, \infty) \times \Omega), \quad \tau \in L^{2}((0, \infty) \times \Omega)
$$

be the solution of 1.2 in the sense of Result $B$ associated to the initial data $u_{0}$ and $A_{0}$.

Then, there exists $0<T^{*}<\infty$ independent of $W e, u^{0}$ a global weak solution of (1.6) associated to the initial data $u_{0}$ and a corrector $A^{1}$, such that, in addition, $u^{0}$ belongs to $L^{\infty}\left((0, T) ; H^{4}\right)$ and $A^{1} \in L^{\infty}\left((0, T) ; H^{3}\right)$ for all $0<T<T^{*}$.

Furthermore, $u$ and $A$ converge in the modulated energy norm

$$
\begin{aligned}
& \sup _{t \in[0, T]}\left(\frac{1}{2}\left\|u-u^{0}\right\|_{L^{2}}^{2}+(1-\omega) \int_{0}^{t}\left\|\nabla\left(u-u^{0}\right)\right\|_{L^{2}}^{2}\right. \\
+ & \left.\frac{\omega(b+d)}{2 b} \frac{1}{W e}\left\|A-A^{0}-W e A^{1}\right\|_{L^{2}}^{2}+\frac{\omega(b+d)}{2 b} \frac{1}{W e^{2}} \int_{0}^{t}\left\|A-A^{0}-W e A^{1}\right\|_{L^{2}}^{2}\right)^{\frac{1}{2}}=O(\sqrt{W e}),
\end{aligned}
$$

on every time interval $[0, T]$, with $0<T<T^{*}$

Notice that the estimate $(1.14)$ implies immediately the following strong convergences:

$$
\begin{aligned}
\left\|u-u^{0}\right\|_{L^{\infty}\left((0, T) ; L^{2}\right)} & =O(\sqrt{W e}), \\
\left\|u-u^{0}\right\|_{L^{2}\left((0, T) ; H^{1}\right)} & =O(\sqrt{W e}), \\
\left\|A-A^{0}\right\|_{L^{\infty}\left((0, T) ; L^{2}\right)} & =O(W e), \\
\left\|A-A^{0}-W e A^{1}\right\|_{L^{2}\left((0, T) ; L^{2}\right)} & =O\left(W e^{3 / 2}\right), \\
\left\|\tau-\tau^{0}\right\|_{L^{1}\left((0, T) ; L^{1}\right)} & =O(\sqrt{W e}) .
\end{aligned}
$$

Most of the remarks made after the strong convergence Theorem 1.3 have their counterpart for the FENE-P system. Let us make a few further comments:

- Notice that 1.13 imposes that

$$
\left\|A_{0}-A^{0}\right\|_{L^{2}(\Omega)}^{2}=O(W e) .
$$

However, looking at the decomposition (3.9) of $\tau$, we note that nothing is prescribed on the second term in the right hand side, namely

$$
\frac{(b+d) \omega}{b} \frac{1}{W e} A \frac{\operatorname{Tr} A-\operatorname{Tr} A^{0}}{b\left(1-\frac{\operatorname{Tr} A^{0}}{b}\right)\left(1-\frac{\operatorname{Tr} A}{b}\right)},
$$

which means that our result holds for ill-prepared data $\tau$, such that $\tau_{0} \neq 2 \omega D\left(u_{0}\right)$. Hence, Theorem 1.4 is in the same spirit as our strong convergence result on the corotational system, and the work of L. Molinet and R. Talhouk [27] on the convergence of strong solutions to the generic Johnson-Segalman model. 
- We carry out the proof in the case $d=3$. The case $d=2$ is even simpler. As in the proof of Theorem 1.3 the restriction on the dimension comes from the estimate of the term

$$
-\int_{0}^{t} \int_{\Omega}\left(\left(u-u^{0}-W e u^{1}\right) \cdot \nabla u^{0}\right) \cdot\left(u-u^{0}-W e u^{1}\right) .
$$

- Note that the smoothness assumption $u_{0} \in H^{4}$ is enough in the case $d=2$ as well as $d=3$. It implies $A^{1} \in L^{\infty}\left((0, T) ; L^{p}\right)$, for all $1 \leq p \leq \infty$.

- The control of the norm 1.14 is obtained through a Taylor expansion of the free energy 1.5 around the corrector $A^{0}+W e A^{1}$. This leads to the study of the positive quantity (3.17), called the relative entropy, which bounds the norm (1.14). Expanding the free energy around $A^{0}$ is not enough. It would yield a bound on $A-A^{0}$, rather than on $A-A^{0}-W e A^{1}$.

After the proof of this theorem in Section 3.2, we comment on the strong convergence for other macroscopic models of viscoelastic fluid flows, namely the Giesekus, the PTT and Oldroyd-B models.

1.3. Organization of the paper. For the reader's convenience we devote the first section of this paper (Section 2) to the proof of the results concerning the corotational system. The proofs are easier in this case, and shed some light on some features, which help to understand our analysis of the FENE-P system. We show the weak convergence of Proposition 1.1 in the Section 2.1 and the strong convergence of Theorem 1.3 in Section 2.2. In Section 3 we address the results related to the FENE-P system. Proposition 1.2 (weak convergence) is showed in Section 3.1 and we demonstrate our Theorem 1.4 (strong convergence) in Section 3.2. At the end of this part, we make some brief comments on the convergence for the Giesekus and PTT models. The proof of Proposition $1.1 \mathrm{bis}$ is postponed to the Appendix A. In this appendix, we also show refined $L^{p}\left((0, T) ; L^{q}\right)$ a priori estimates on the corotational system. These estimates are also the key for the existence of weak solutions to the corotational system.

2. Low Weissenberg limit for the corotational system. We concentrate on the low Weissenberg asymptotic analysis of the corotational system (1.1). The first part of this section is devoted to the weak convergence. In the second subsection, we show the strong convergence result of Theorem 1.3 relying on a relative entropy method.

2.1. Weak convergence. We carry out the proof of Proposition 1.1 in the case when $\Omega \subset \mathbb{R}^{d}$ is a bounded domain and $d=2,3$. Our analysis extends straightforwardly to the case $\Omega=\mathbb{T}^{d}$, and $\Omega=\mathbb{R}^{d}$, as we only work with local in space bounds. Let $(u, \tau)$ be a sequence of weak solutions to (1.1) satisfying the a priori bound (1.3). According to the assumption (1.7) on the initial data, we deduce that

$$
\begin{aligned}
& u \text { is uniformly bounded in } W e \text { in } L^{\infty}\left((0, \infty) ; L^{2}\right) \cap L^{2}\left((0, \infty) ; \dot{H}^{1}\right), \\
& \tau \quad L^{2}\left((0, \infty) ; L^{2}\right) .
\end{aligned}
$$

Let us notice that the bound on $\tau$ in $L^{\infty}\left((0, \infty) ; L^{2}\right)$ is not uniform in $W e$.

Compactness. As is usual, the former bounds imply the existence of

$$
u^{0} \in L^{\infty}\left((0, \infty) ; L^{2, \sigma}\right) \cap L^{2}\left((0, \infty) ; \dot{H}^{1}\right) \quad \text { and } \quad \tau^{0} \in L^{2}\left((0, \infty) ; L^{2}\right),
$$

such that the following convergences hold (extracting subsequences if necessary), for all $0<T<\infty$,

$$
\begin{aligned}
& u \rightarrow u^{0} \quad L^{2}\left((0, T) ; H^{1}\right), \\
& u(t, \cdot) \rightarrow u^{0}(t, \cdot) \quad L^{2}(\Omega), \\
& u \stackrel{*}{\rightarrow} u^{0} \quad L^{\infty}\left((0, \infty) ; L^{2}\right), \\
& \nabla u \rightarrow \nabla u^{0} \quad L^{2}\left((0, \infty) ; L^{2}\right), \\
& \tau \rightarrow \tau^{0} \quad L^{2}\left((0, \infty) ; L^{2}\right), \\
& \tau(t, \cdot) \rightarrow \tau^{0}(t, \cdot) \quad L^{2}(\Omega),
\end{aligned}
$$


and

$$
\partial_{t} u \quad \text { is uniformly bounded in } L^{4 / d}\left((0, T) ; V^{\prime}\right) \text {, }
$$

where $V^{\prime}$ is the dual of $V:=\left\{v \in H_{0}^{1}, \nabla \cdot v=0\right\}$. For the latter bound we note that $\nabla \cdot \tau$ is bounded in $L^{2}\left((0, T) ; H^{-1}\right)$. The non-linear term is the only tricky one to estimate: for all $\varphi_{\sigma} \in C_{c}^{\infty}\left((0, \infty) ; C_{c}^{\infty, \sigma}\right)$, for all $0<t<T$,

$$
\left|\left\langle-u \cdot \nabla u(t, \cdot), \varphi_{\sigma}(t, \cdot)\right\rangle_{\mathcal{D}^{\prime}, \mathcal{D}}\right| \leq\|u(t, \cdot)\|_{L^{2}}^{2-d / 2}\|u(t, \cdot)\|_{H^{1}}^{d / 2}\left\|\varphi_{\sigma}(t, \cdot)\right\|_{V} .
$$

Therefore, using the Aubin-Lions lemma [5], we get the strong convergence

$$
u \longrightarrow u^{0} \quad L^{2}\left((0, T) ; L^{2}\right) .
$$

Weak convergence of $\tau$ and $u$. The simple observation leading to the convergence of $\tau$ is that

$$
2 \omega D(u)-\tau=W e\left(\partial_{t} \tau+u \cdot \nabla \tau+\tau W(u)-W(u) \tau\right) .
$$

The Weissenberg number in front of the right hand side makes the convergence follow directly from the bounds on $u$ and $\tau$ above. The Newtonian limit is therefore much more simple than passing to the limit on a sequence of approximated solutions, when proving the existence of weak solutions. We do not need refined convergence results for $\tau$. For all $\psi \in C_{c}^{\infty}((0, \infty) \times \Omega)$,

$$
\begin{aligned}
& \langle 2 \omega D(u)-\tau, \psi\rangle_{\mathcal{D}^{\prime}, \mathcal{D}} \\
& =\left\langle\partial_{t} \tau+u \cdot \nabla \tau+\tau W(u)-W(u) \tau, \psi\right\rangle_{\mathcal{D}^{\prime}, \mathcal{D}} \\
& =W e\left[-\left\langle\tau, \partial_{t} \psi+u \cdot \nabla \psi\right\rangle_{L^{2}, L^{2}}+\langle\tau W(u)-W(u) \tau, \psi\rangle_{\mathcal{D}^{\prime}, \mathcal{D}}\right] \stackrel{W e \rightarrow 0}{\longrightarrow} 0 .
\end{aligned}
$$

Moreover,

$$
\langle 2 \omega D(u)-\tau, \psi\rangle_{\mathcal{D}^{\prime}, \mathcal{D}} \stackrel{W e \rightarrow 0}{\longrightarrow}\left\langle 2 \omega D\left(u^{0}\right)-\tau^{0}, \psi\right\rangle_{\mathcal{D}^{\prime}, \mathcal{D}}
$$

which yields at the limit, $\tau^{0}=2 \omega D\left(u^{0}\right)$.

Let $\varphi_{\sigma} \in C_{c}^{\infty}\left((0, \infty) ; C_{c}^{\infty, \sigma}\right)$. The strong convergence of the velocity field in $L^{2}\left((0, T) ; L^{2}\right)$ allows to pass to the weak limit in the nonlinear term $u \cdot \nabla u$. Hence, we can pass to the limit in the momentum equation and get that $u^{0}$ satisfies

$$
\left\langle\partial_{t} u^{0}+u^{0} \cdot \nabla u^{0}-(1-\omega) \Delta u^{0}, \varphi_{\sigma}\right\rangle_{\mathcal{D}^{\prime}, \mathcal{D}}=\left\langle\tau^{0}, \varphi_{\sigma}\right\rangle_{\mathcal{D}^{\prime}, \mathcal{D}}=\omega\left\langle\Delta u^{0}, \varphi_{\sigma}\right\rangle_{\mathcal{D}^{\prime}, \mathcal{D}} .
$$

It remains to apply De Rham's theorem (see for example [35] for a rigorous statement) to ensure that $u^{0}$ is a weak solution to the Navier-Stokes system (1.6).

REMARK 1. We can pass to the limit in the inequality (1.3) assuming furthermore that $\sqrt{W e} \tau_{0}$ tends to zero in $L^{2}$. Then using that $\tau^{0}=2 \omega D\left(u^{0}\right)$ and the obtained weak convergences, we get the standard energy inequality related to the Navier-Stokes equations.

2.2. Strong convergence. Our goal is to give a proof of Theorem 1.3 stating the strong convergence of the velocity field $u$ and of the symmetric stress tensor $\tau$ of the fluid flow solving (1.1. . Our modus operandi emphasizes in a simple case some features of the relative entropy method used for the strong convergence in the FENE-P system.

To get the strong convergence, we expand $u, p$ and $\tau$ in powers of $W e$ :

$$
u \simeq u^{0}+W e u^{1}, \quad p \simeq p^{0}+W e p^{1}, \quad \tau \simeq \tau^{0}+W e \tau^{1} .
$$

Very formal computations yield, as expected, that the lower order term $u^{0}=u^{0}(t, x) \in \mathbb{R}^{3}$ should solve the three-dimensional Navier-Stokes equation

$$
\left\{\begin{array}{rl}
\partial_{t} u^{0}+u^{0} \cdot \nabla u^{0}-\Delta u^{0}+\nabla p^{0} & =0 \\
\nabla \cdot u^{0} & =0
\end{array},\right.
$$


and that the stress tensor

$$
\tau^{0}=2 \omega D\left(u^{0}\right)
$$

At first order in $W e$, we expect $u^{1}=u^{1}(t, x) \in \mathbb{R}^{3}$ and the symmetric tensor $\tau^{1}=\tau^{1}(t, x) \in \mathbb{R}^{3}$ to solve

$$
\left\{\begin{array}{rl}
\partial_{t} u^{1}+u^{0} \cdot \nabla u^{1}+u^{1} \cdot \nabla u^{0}-(1-\omega) \Delta u^{1}+\nabla p^{1} & =\nabla \cdot \tau^{1} \\
\nabla \cdot u^{1} & =0 \\
\partial_{t} \tau^{0}+u^{0} \cdot \nabla \tau^{0}+\tau^{0} W\left(u^{0}\right)-W\left(u^{0}\right) \tau^{0}+\tau^{1} & =2 \omega D\left(u^{1}\right)
\end{array} .\right.
$$

We aim at showing, first in the case of $\Omega=\mathbb{R}^{3}$ that these expansions are in fact correct, as well for well-prepared as for ill-prepared data $\tau_{0}$. The idea of the proof is classical and consists in using the relative entropy of the viscoelastic system. We proceed in two steps for the proof: first we show the well-posedness of system (2.4), then we prove a Gronwall type inequality on the relative entropy.

2.2.1. On the necessity of the first-order correctors. The first-order correctors $u^{1}$ and $\tau^{1}$ are needed to achieve our estimates, although there are transparent in the final convergence result 1.12 . Indeed, if one stops the expansion of $u$ and $\tau$ at order 0 , we get the estimate:

$$
\begin{array}{r}
\omega\left\|u(t, \cdot)-u^{0}(t, \cdot)\right\|_{L^{2}}^{2}+2 \omega(1-\omega) \int_{0}^{t}\left\|\nabla\left(u-u^{0}\right)\right\|_{L^{2}}^{2}+\frac{W e}{2}\|\tau(t, \cdot)\|_{L^{2}}^{2} \\
+\int_{0}^{t}\left\|\tau-2 \omega D\left(u^{0}\right)\right\|_{L^{2}}^{2} \leq \frac{W e}{2}\left\|\tau_{0}\right\|_{L^{2}}^{2}-2 \omega \int_{0}^{t} \int_{\mathbb{R}^{3}}\left(\left(u-u^{0}\right) \cdot \nabla u^{0}\right) \cdot\left(u-u^{0}\right) \\
-2 \omega \int_{0}^{t} \int_{\mathbb{R}^{3}}(\tau-2 \omega D(u)): D\left(u^{0}\right),
\end{array}
$$

which does not seem to allow to conclude. In fact, the natural idea would be to bound

$$
\left|-2 \omega \int_{0}^{t} \int_{\mathbb{R}^{3}}\left(\left(u-u^{0}\right) \cdot \nabla u^{0}\right) \cdot\left(u-u^{0}\right)\right| \leq 2 \omega \int_{0}^{t}\left\|\nabla u^{0}\right\|_{L^{\infty}}\left\|u-u^{0}\right\|_{L^{2}}^{2},
$$

and to split

$$
\begin{array}{r}
-2 \omega \int_{0}^{t} \int_{\mathbb{R}^{3}}(\tau-2 \omega D(u)): D\left(u^{0}\right)=-2 \omega \int_{0}^{t} \int_{\mathbb{R}^{3}}\left(\tau-2 \omega D\left(u^{0}\right)\right): D\left(u^{0}\right) \\
\quad-4 \omega^{2} \int_{0}^{t} \int_{\mathbb{R}^{3}}\left(D\left(u^{0}\right)-D(u)\right): D\left(u^{0}\right) .
\end{array}
$$

The term in the right hand side of 2.6 is nice since $\left\|\nabla u^{0}\right\|_{L^{\infty}}$ is locally integrable in time and can be dealt with using Gronwall's inequality. The annoying terms are the ones appearing in (2.7). We can bound the latter by

$$
\begin{aligned}
2 \omega \int_{0}^{t} \| \tau-2 \omega D\left(u^{0}\right) & \left\|_{L^{2}}\right\| D\left(u^{0}\right)\left\|_{L^{2}}+4 C \omega^{2} \int_{0}^{t}\right\| \nabla\left(u-u^{0}\right)\left\|_{L^{2}}\right\| D\left(u^{0}\right) \|_{L^{2}} \\
\leq C & {\left[\nu \int_{0}^{t}\left\|\tau-2 \omega D\left(u^{0}\right)\right\|_{L^{2}}^{2}+\nu \int_{0}^{t}\left\|\nabla\left(u-u^{0}\right)\right\|_{L^{2}}^{2}+\frac{1}{\nu} \int_{0}^{t}\left\|D\left(u^{0}\right)\right\|_{L^{2}}^{2}\right] }
\end{aligned}
$$

and absorb some terms for $\nu$ small in the left hand side of 2.5 . Yet, the term

$$
\frac{1}{\nu} \int_{0}^{t}\left\|D\left(u^{0}\right)\right\|_{L^{2}}^{2}
$$

remaining in the right hand side need not to be small in the limit $W e \rightarrow 0$. 
2.2.2. Well-posedness of the profiles. We carry out an $H^{m}$ a priori estimate on $(2.2)$ in the same fashion as was done in [36] for the Euler system: there exists $C>0$ such that, for $m \geq 3$, for all $t$ sufficiently small,

$$
\frac{1}{2}\left\|u^{0}(t, \cdot)\right\|_{H^{m}}^{2}+\int_{0}^{t}\left\|\nabla u^{0}(s, \cdot)\right\|_{H^{m}}^{2} d s \leq \frac{1}{2}\left\|u_{0}\right\|_{H^{m}}^{2}+C_{0}\left\|u^{0}(t, \cdot)\right\|_{H^{m}}^{3} .
$$

Hence, for all $0<T<\frac{1}{C_{0}\left\|u_{0}\right\|_{H^{m}}}=: T^{*}$,

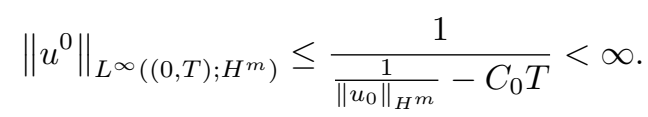

Therefore, there exists a global weak solution $u^{0} \in C^{0}\left([0, \infty) ; H^{-1}\right) \cap L^{\infty}\left((0, \infty) ; L^{2, \sigma}\right) \cap L^{2}\left((0, \infty) ; \dot{H}^{1}\right)$ of (2.2), such that, for all $0<T<T^{*}, u^{0} \in L^{\infty}\left((0, T) ; H^{m}\right)$. This regularity for $m=4$ is sufficient for the rest of the computations. Note that an $L^{2}\left((0, T) ; H^{4}\right)$ bound on $u^{0}$ is not enough to us, so that we do not take advantage of the regularizing effect of $(2.2)$ to weaken the assumption on the initial data $u_{0}$.

From (2.4), one retrieves

$$
\tau^{1}=2 \omega D\left(u^{1}\right)-\partial_{t} \tau^{0}-u^{0} \cdot \nabla \tau^{0}-\tau^{0} W\left(u^{0}\right)+W\left(u^{0}\right) \tau^{0},
$$

so that we can introduce it in the momentum equation at first order:

$$
\left\{\begin{array}{rl}
\partial_{t} u^{1}+u^{0} \cdot \nabla u^{1}+u^{1} \cdot \nabla u^{0}-\Delta u^{1}+\nabla p^{1} & =f^{1} \\
\nabla \cdot u^{1} & =0
\end{array} .\right.
$$

We complement 2.10 with the initial data $u^{1}(0, \cdot)=0$. Using the equation satisfied by $u^{0}$, the source term may be written under the form

$$
\begin{aligned}
f^{1}:=-\partial_{t}\left(\nabla \cdot \tau^{0}\right)-\nabla \cdot\left(u^{0} \cdot \nabla \tau^{0}\right)-\nabla \cdot\left(\tau^{0} W\left(u^{0}\right)\right)+\nabla \cdot\left(W\left(u^{0}\right) \tau^{0}\right) \\
=\omega \Delta\left(u^{0} \cdot \nabla u^{0}\right)-\omega \Delta^{2} u^{0}+\nabla \Delta p^{0}-2 \omega \nabla \cdot\left(u^{0} \cdot \nabla D\left(u^{0}\right)\right)-2 \omega \nabla \cdot\left(D\left(u^{0}\right) W\left(u^{0}\right)\right) \\
\quad+2 \omega \nabla \cdot\left(W\left(u^{0}\right) D\left(u^{0}\right)\right) \\
=\omega \Delta\left(u^{0} \cdot \nabla u^{0}\right)-\omega \Delta^{2} u^{0}-\nabla \nabla \cdot\left(u^{0} \cdot \nabla u^{0}\right)-2 \omega \nabla \cdot\left(u^{0} \cdot \nabla D\left(u^{0}\right)\right) \\
\quad-2 \omega \nabla \cdot\left(D\left(u^{0}\right) W\left(u^{0}\right)\right)+2 \omega \nabla \cdot\left(W\left(u^{0}\right) D\left(u^{0}\right)\right)
\end{aligned}
$$

which is in $L^{\infty}\left((0, T) ; L^{2}\right)$. Straightforward energy estimates on 2.10 show that a sequence of approximated solutions is bounded in $L^{\infty}\left((0, T) ; L^{2}\right) \cap L^{2}\left((0, T) ; H^{1}\right)$, which yields the existence of a weak solution

$$
u^{1} \in C^{0}\left([0, T) ; H^{-1}\right) \cap L^{\infty}\left((0, T) ; L^{2, \sigma}\right) \cap L^{2}\left((0, T) ; H^{1}\right) .
$$

Using the regularity of $u^{0}$, we get the extra estimate $u^{1} \in L^{\infty}\left((0, T) ; H^{1}\right)$. Hence, one deduces from the latter, (2.9) and the equation satisfied by $u^{0}$, that $\tau^{1} \in L^{2}\left((0, T) ; L^{2}\right)$.

2.2.3. Weak strong estimate. We now turn to the estimation of the remainders

$$
U^{(r)}:=u-u^{0}-W e u^{1}
$$

and $\tau-\tau^{0}$. In order to carry out the computations below, we need the regularity on the profiles $u^{0}, u^{1}, \tau^{0}$ and $\tau^{1}$ we have assumed above. On the one hand

$$
\begin{aligned}
\partial_{t} U^{(r)}+U^{(r)} \cdot \nabla u+u^{0} \cdot \nabla U^{(r)}-(1-\omega) \Delta U^{(r)}+\nabla\left(p-p^{0}-W e p^{1}\right) & \\
& =-W e\left(u^{1} \cdot \nabla\left(u-u^{0}\right)\right)+\nabla \cdot\left(\tau-\tau^{0}-W e \tau^{1}\right),
\end{aligned}
$$

which yields

$$
\begin{aligned}
\frac{1}{2}\left\|U^{(r)}(t, \cdot)\right\|_{L^{2}}^{2} & +(1-\omega) \int_{0}^{t}\left\|\nabla U^{(r)}\right\|_{L^{2}}^{2}+\int_{0}^{t} \int_{\mathbb{R}^{3}}\left(U^{(r)} \cdot \nabla u\right) \cdot U^{(r)} \\
& \leq-W e \int_{0}^{t} \int_{\mathbb{R}^{3}}\left(u^{1} \cdot \nabla\left(u-u^{0}\right)\right) \cdot U^{(r)}-\int_{0}^{t} \int_{\mathbb{R}^{3}}\left(\tau-\tau^{0}-W e \tau^{1}\right): \nabla U^{(r)} .
\end{aligned}
$$


On the other hand

$$
\begin{array}{r}
\partial_{t}\left(\tau-\tau^{0}\right)+u \cdot \nabla\left(\tau-\tau^{0}\right)+\frac{\tau-\tau^{0}}{W e}=-\left(u-u^{0}\right) \cdot \nabla \tau^{0}-\left(\tau-\tau^{0}\right) W(u)-\tau^{0}\left(W(u)-W\left(u^{0}\right)\right) \\
+W(u)\left(\tau-\tau^{0}\right)+\left(W(u)-W\left(u^{0}\right)\right) \tau^{0}+\tau^{1}+2 \omega \frac{D\left(U^{(r)}\right)}{W e}
\end{array}
$$

which gives

$$
\begin{aligned}
& \frac{1}{2}\left\|\tau(t, \cdot)-\tau^{0}(t, \cdot)\right\|_{L^{2}}^{2}+\frac{1}{W e} \int_{0}^{t}\left\|\tau-\tau^{0}\right\|_{L^{2}}^{2}=\frac{1}{2}\left\|\tau_{0}-2 \omega D\left(u_{0}\right)\right\|_{L^{2}}^{2} \\
& \quad+\int_{0}^{t} \int_{\mathbb{R}^{3}} \tau^{1}:\left(\tau-\tau^{0}\right)-\int_{0}^{t} \int_{\mathbb{R}^{3}}\left(\left(u-u^{0}\right) \cdot \nabla \tau^{0}\right):\left(\tau-\tau^{0}\right) \\
& \quad+\int_{0}^{t} \int_{\mathbb{R}^{3}}\left(\tau^{0} W\left(u-u^{0}\right)\right):\left(\tau-\tau^{0}\right)+\int_{0}^{t} \int_{\mathbb{R}^{3}}\left(W\left(u^{0}-u\right) \tau^{0}\right):\left(\tau-\tau^{0}\right) \\
& \quad+\frac{2 \omega}{W e} \int_{0}^{t} \int_{\mathbb{R}^{3}} \nabla U^{(r)}:\left(\tau-\tau^{0}\right) .
\end{aligned}
$$

The linear combination $2 \omega(2.12)+W e(2.13)$ gives the energy equality

$$
\begin{aligned}
& \omega\left\|U^{(r)}(t, \cdot)\right\|_{L^{2}}^{2}+2 \omega(1-\omega) \int_{0}^{t}\left\|\nabla U^{(r)}\right\|_{L^{2}}^{2}+\frac{W e}{2}\left\|\tau(t, \cdot)-\tau^{0}(t, \cdot)\right\|_{L^{2}}^{2}+\int_{0}^{t}\left\|\tau-\tau^{0}\right\|_{L^{2}}^{2} \\
& \leq \frac{W e}{2}\left\|\tau_{0}-2 \omega D\left(u_{0}\right)\right\|_{L^{2}}^{2}-2 \omega \int_{0}^{t} \int_{\mathbb{R}^{3}}\left(U^{(r)} \cdot \nabla u\right) \cdot U^{(r)} \\
& \quad-2 \omega W e \int_{0}^{t} \int_{\mathbb{R}^{3}}\left(u^{1} \cdot \nabla\left(u-u^{0}\right)\right) \cdot U^{(r)}+W e \int_{0}^{t} \int_{\mathbb{R}^{3}} \tau^{1}:\left(\tau-\tau^{0}\right) \\
& \quad-W e \int_{0}^{t} \int_{\mathbb{R}^{3}}\left(\left(u-u^{0}\right) \cdot \nabla \tau^{0}\right):\left(\tau-\tau^{0}\right)+W e \int_{0}^{t} \int_{\mathbb{R}^{3}}\left(\tau^{0} W\left(u-u^{0}\right)\right):\left(\tau-\tau^{0}\right) \\
& \left.\quad+W e \int_{0}^{t} \int_{\mathbb{R}^{3}}\left(W\left(u^{0}-u\right) \tau^{0}\right):\left(\tau-\tau^{0}\right)+4 \omega^{2} W e \int_{0}^{t} \int_{\mathbb{R}^{3}} \tau^{1}: \nabla U^{(r)}\right) \\
& =\frac{W e}{2}\left\|\tau_{0}-2 \omega D\left(u_{0}\right)\right\|_{L^{2}}^{2}+\int_{0}^{t}(\mathrm{~A}+\mathrm{B}+\mathrm{C}+\mathrm{D}+\mathrm{E}+\mathrm{F}+\mathrm{G}) .
\end{aligned}
$$

We estimate each term of the right hand side of 2.15 separately. The goal is to split each term into a part which is sufficiently small to be absorbed by the left hand side of $(2.15)$, a part which is controlled through a Gronwall type inequality and remainder terms of order $O(W e)$. Let $\nu>0$. This parameter is going to be taken small independently of $0<W e<1$ in the sequel. We have

$$
\begin{aligned}
|\mathrm{A}| & \leq 2 \omega\left|\int_{\mathbb{R}^{3}}\left(U^{(r)} \cdot \nabla u^{0}\right) \cdot U^{(r)}\right|+2 \omega W e\left|\int_{\mathbb{R}^{3}}\left(U^{(r)} \cdot \nabla u^{1}\right) \cdot U^{(r)}\right| \\
& \leq 2 \omega\left\|U^{(r)}\right\|_{L^{3}}\left\|u^{0}\right\|_{L^{6}}\left\|\nabla U^{(r)}\right\|_{L^{2}}+2 \omega W e\left\|U^{(r)}\right\|_{L^{3}}\left\|u^{1}\right\|_{L^{6}}\left\|\nabla U^{(r)}\right\|_{L^{2}} \\
& \leq 2 \omega \nu^{-\frac{3}{4}}\left\|U^{(r)}\right\|_{L^{2}}^{\frac{1}{2}}\left\|\nabla u^{0}\right\|_{L^{2}} \nu^{\frac{3}{4}}\left\|\nabla U^{(r)}\right\|_{L^{2}}^{\frac{3}{2}}+2 \omega W e\left\|U^{(r)}\right\|_{L^{2}}^{\frac{1}{2}}\left\|\nabla u^{1}\right\|_{L^{2}}\left\|\nabla U^{(r)}\right\|_{L^{2}}^{\frac{3}{2}} \\
& \leq \frac{\omega}{2 \nu^{3}}\left\|U^{(r)}\right\|_{L^{2}}^{2}\left\|\nabla u^{0}\right\|_{L^{2}}^{4}+\frac{3 \omega \nu}{2}\left\|\nabla U^{(r)}\right\|_{L^{2}}^{2} \\
& \quad+\frac{\omega}{2 \nu^{3}} W e\left\|U^{(r)}\right\|_{L^{2}}^{2}\left\|\nabla u^{1}\right\|_{L^{2}}^{4}+\frac{3 \omega \nu}{2} W e\left\|\nabla U^{(r)}\right\|_{L^{2}}^{2} .
\end{aligned}
$$


The second term is of order $O\left(W e^{2}\right)$. Indeed,

$$
\begin{aligned}
|\mathrm{B}| & \leq 2 \omega W e\left|\int_{\mathbb{R}^{3}}\left(u^{1} \cdot \nabla U^{(r)}\right) \cdot U^{(r)}\right|+2 \omega W e^{2}\left|\int_{\mathbb{R}^{3}}\left(u^{1} \cdot \nabla u^{1}\right) \cdot U^{(r)}\right| \\
& =2 \omega W e^{2}\left|\int_{\mathbb{R}^{3}}\left(u^{1} \cdot \nabla u^{1}\right) \cdot U^{(r)}\right| \\
& \leq 2 \omega W e^{2}\left\|u^{1}\right\|_{L^{3}}\left\|u^{1}\right\|_{L^{6}}\left\|\nabla U^{(r)}\right\|_{L^{2}} \leq 2 \omega W e^{2}\left\|u^{1}\right\|_{L^{2}}^{\frac{1}{2}}\left\|\nabla u^{1}\right\|_{L^{2}}^{\frac{3}{2}}\left\|\nabla U^{(r)}\right\|_{L^{2}} \\
& \leq \frac{\omega}{\nu} W e^{2}\left\|u^{1}\right\|_{L^{2}}\left\|\nabla u^{1}\right\|_{L^{2}}^{3}+\omega \nu W e^{2}\left\|\nabla U^{(r)}\right\|_{L^{2}}^{2} .
\end{aligned}
$$

The third term is estimated in a simple way

$$
|\mathrm{C}| \leq W e\left\|\tau^{1}\right\|_{L^{2}}\left\|\tau-\tau^{0}\right\|_{L^{2}} \leq \frac{W e}{2}\left\|\tau^{1}\right\|_{L^{2}}^{2}+\frac{W e}{2}\left\|\tau-\tau^{0}\right\|_{L^{2}}^{2}
$$

so is the last term

$$
|\mathrm{G}| \leq \frac{2 \omega^{2}}{\nu} W e\left\|\tau^{1}\right\|_{L^{2}}^{2}+2 \omega^{2} \nu W e\left\|\nabla U^{(r)}\right\|_{L^{2}}^{2}
$$

For the fourth term, we rely again on a convexity inequality

$$
\begin{aligned}
|\mathrm{D}| & \leq W e\left|\int_{\mathbb{R}^{3}}\left(U^{(r)} \cdot \nabla \tau^{0}\right):\left(\tau-\tau^{0}\right)\right|+W e^{2}\left|\int_{\mathbb{R}^{3}}\left(u^{1} \cdot \nabla \tau^{0}\right):\left(\tau-\tau^{0}\right)\right| \\
& \leq W e\left\|U^{(r)}\right\|_{L^{2}}\left\|\nabla \tau^{0}\right\|_{L^{\infty}}\left\|\tau-\tau^{0}\right\|_{L^{2}}+W e^{2}\left\|u^{1} \cdot \nabla \tau^{0}\right\|_{L^{2}}\left\|\tau-\tau^{0}\right\|_{L^{2}} \\
& \leq \frac{W e}{2}\left\|U^{(r)}\right\|_{L^{2}}^{2}\left\|\nabla \tau^{0}\right\|_{L^{\infty}}^{2}+\frac{W e}{2}\left\|\tau-\tau^{0}\right\|_{L^{2}}^{2} \\
& +\frac{W e^{2}}{2}\left\|u^{1}\right\|_{L^{2}}^{2}\left\|\nabla \tau^{0}\right\|_{L^{\infty}}^{2}+\frac{W e^{2}}{2}\left\|\tau-\tau^{0}\right\|_{L^{2}}^{2} .
\end{aligned}
$$

The next two terms are treated analogously:

$$
\begin{aligned}
|\mathrm{E}| & \leq W e\left|\int_{\mathbb{R}^{3}}\left(\tau^{0} W\left(U^{(r)}\right)\right):\left(\tau-\tau^{0}\right)\right|+W e^{2}\left|\int_{\mathbb{R}^{3}}\left(\tau^{0} W\left(u^{1}\right)\right):\left(\tau-\tau^{0}\right)\right| \\
\leq & W e\left\|\tau^{0}\right\|_{L^{\infty}}\left\|\nabla U^{(r)}\right\|_{L^{2}}\left\|\tau-\tau^{0}\right\|_{L^{2}}+W e^{2}\left\|\tau^{0}\right\|_{L^{\infty}}\left\|\nabla u^{1}\right\|_{L^{2}}\left\|\tau-\tau^{0}\right\|_{L^{2}} \\
\leq & \frac{W e \nu}{2}\left\|\tau^{0}\right\|_{L^{\infty}}^{2}\left\|\nabla U^{(r)}\right\|_{L^{2}}^{2}+\frac{W e}{2 \nu}\left\|\tau-\tau^{0}\right\|_{L^{2}}^{2} \\
& \quad+\frac{W e^{2}}{2}\left\|\tau^{0}\right\|_{L^{\infty}}^{2}\left\|\nabla u^{1}\right\|_{L^{2}}^{2}+\frac{W e^{2}}{2}\left\|\tau-\tau^{0}\right\|_{L^{2}},
\end{aligned}
$$

and the same type of estimate holds for F. We deduce from these estimates that there exists a value of $\nu>0$, depending (among others) on $\omega$ and $\left\|u_{0}\right\|_{H^{4}(\Omega)}$, but not on $W e$, such that for all $0<W e<1$

$$
\begin{aligned}
& \omega\left\|U^{(r)}(t, \cdot)\right\|_{L^{2}}^{2}+\omega(1-\omega) \int_{0}^{t}\left\|\nabla U^{(r)}\right\|_{L^{2}}^{2}+\frac{W e}{2}\left\|\tau(t, \cdot)-\tau^{0}(t, \cdot)\right\|_{L^{2}}^{2}+\frac{1}{2} \int_{0}^{t}\left\|\tau-\tau^{0}\right\|_{L^{2}}^{2} \\
& \leq \frac{W e}{2}\left\|\tau_{0}-2 \omega D\left(u_{0}\right)\right\|_{L^{2}}^{2} \\
& \quad+C_{\nu} \int_{0}^{t}\left(\left\|\nabla u^{0}\right\|_{L^{2}}^{4}+W e\left\|\nabla \tau^{0}\right\|_{L^{\infty}}^{2}+W e\left\|\nabla u^{1}\right\|_{L^{2}}^{4}+1\right)\left(\omega\left\|U^{(r)}\right\|_{L^{2}}^{2}+\frac{W e}{2}\left\|\tau-\tau^{0}\right\|_{L^{2}}\right) \\
& \quad+\frac{\omega}{\nu} W e^{2} \int_{0}^{t}\left\|u^{1}\right\|_{L^{2}}\left\|\nabla u^{1}\right\|_{L^{2}}^{3}+\frac{W e}{2} \int_{0}^{t}\left\|\tau^{1}\right\|_{L^{2}}^{2} \\
& \quad+\frac{W e^{2}}{2} \int_{0}^{t}\left\|u^{1}\right\|_{L^{2}}^{2}\left\|\nabla \tau^{0}\right\|_{L^{\infty}}^{2}+\frac{2 \omega^{2}}{\nu} W e \int_{0}^{t}\left\|\tau^{1}\right\|_{L^{2}}^{2}+\frac{W e^{2}}{2} \int_{0}^{t}\left\|\tau^{0}\right\|_{L^{\infty}}^{2}\left\|\nabla u^{1}\right\|_{L^{2}}^{2} .
\end{aligned}
$$


The constant $C_{\nu}$ depends on the choice of $\nu$, on $\omega$ and again on $\left\|u_{0}\right\|_{H^{4}(\Omega)}$, but is independent of $W e$. Via Gronwall's lemma, we finally manage to control a relative entropy associated to the viscoelastic system (1.1): for all $0 \leq t \leq T<T^{*}$,

$$
\begin{aligned}
\omega & \left\|U^{(r)}(t, \cdot)\right\|_{L^{2}}^{2}+\omega(1-\omega) \int_{0}^{t}\left\|\nabla U^{(r)}\right\|_{L^{2}}^{2}+\frac{W e}{2}\left\|\tau(t, \cdot)-\tau^{0}(t, \cdot)\right\|_{L^{2}}^{2}+\frac{1}{2} \int_{0}^{t}\left\|\tau-\tau^{0}\right\|_{L^{2}}^{2} \\
\leq & W e\left[\frac{1}{2}\left\|\tau_{0}-2 \omega D\left(u_{0}\right)\right\|_{L^{2}}^{2}+\int_{0}^{t}\left(\frac{\omega}{\nu} W e\left\|u^{1}\right\|_{L^{2}}\left\|\nabla u^{1}\right\|_{L^{2}}^{3}\right.\right. \\
& +\frac{1}{2}\left\|\tau^{1}\right\|_{L^{2}}^{2}+\frac{W e}{2}\left\|u^{1}\right\|_{L^{2}}^{2}\left\|\nabla \tau^{0}\right\|_{L^{\infty}}^{2}+\frac{W e^{2}}{2}\left\|\tau^{0}\right\|_{L^{\infty}}^{2}\left\|\nabla u^{1}\right\|_{L^{2}}^{2} \\
& \left.\left.+\frac{2 \omega^{2}}{\nu}\left\|\tau^{1}\right\|_{L^{2}}^{2}\right)\right] \exp \left(t+C_{\nu} \int_{0}^{t}\left(\left\|\nabla u^{0}\right\|_{L^{2}}^{4}+W e\left\|\nabla \tau^{0}\right\|_{L^{\infty}}^{2}+W e\left\|\nabla u^{1}\right\|_{L^{2}}^{4}+1\right)\right) .
\end{aligned}
$$

This estimate shows the convergence statement $(1.12)$ of Theorem 1.3 in the modulated energy norm.

3. Low Weissenberg limit for a FENE-P type fluid. In this section, we focus on the low Weissenberg limit for global weak solutions of $\sqrt{1.2}$ posed in a bounded domain $\Omega \subset \mathbb{R}^{d}$, in the torus $\Omega=\mathbb{T}^{d}$ or in $\mathbb{R}^{d}$.

The free energy 1.5 is the fundamental tool for the mathematical analysis of the FENE-P system. It plays a role analogous to the energy 1.3 of the corotational system. However, due to its non-trivial form, it leads to intricate computations.

Because of its importance, we recall the result of $\mathrm{D}$. Hu and T. Lelièvre [17]

$$
\begin{aligned}
& \frac{1}{2}\|u(t, \cdot)\|_{L^{2}(\Omega)}^{2}+(1-\omega) \int_{0}^{t}\|\nabla u\|_{L^{2}(\Omega)}^{2} \\
& \quad+\frac{\omega(b+d)}{2 b} \frac{1}{W e} \int_{\Omega}\left[-\ln (\operatorname{det} A)-b \ln \left(1-\frac{\operatorname{Tr}(A)}{b}\right)+(b+d) \ln \left(\frac{b}{b+d}\right)\right](t) \\
& \quad+\frac{\omega(b+d)}{2 b} \frac{1}{W e^{2}} \int_{0}^{t} \int_{\Omega}\left[\frac{\operatorname{Tr} A}{\left(1-\frac{\operatorname{Tr} A}{b}\right)^{2}}-\frac{2 d}{\left.1-\frac{\operatorname{TrA}}{b}+\operatorname{Tr}\left(A^{-1}\right)\right]}\right. \\
& \leq \frac{1}{2}\left\|u_{0}\right\|_{L^{2}(\Omega)}^{2}+\frac{\omega(b+d)}{2 b} \frac{1}{W e} \int_{\Omega}\left[-\ln \left(\operatorname{det} A_{0}\right)-b \ln \left(1-\frac{\operatorname{Tr}\left(A_{0}\right)}{b}\right)+(b+d) \ln \left(\frac{b}{b+d}\right)\right]
\end{aligned}
$$

and give an outline of how this a priori estimate is derived. We work with regular solutions of (1.2). The free energy estimate relies on the computation of the total time derivative of the left hand side of (3.1) using the formula: for any invertible matrix $M=M(t)$ depending smoothly on $t$

$$
\left(\partial_{t}+u \cdot \nabla\right)(\ln \operatorname{det} M)=\operatorname{Tr}\left(M^{-1}\left(\partial_{t}+u \cdot \nabla\right) M\right) .
$$

A simple energy estimate yields on the one hand,

$$
\begin{aligned}
\frac{1}{2}\|u(t, \cdot)\|_{L^{2}}+(1-\omega) \int_{0}^{t}\|\nabla u(t, \cdot)\|_{L^{2}} & =-\int_{0}^{t} \int_{\Omega} \tau: \nabla u+\frac{1}{2}\left\|u_{0}\right\|_{L^{2}} \\
& =-\frac{1}{W e} \frac{(b+d) \omega}{b} \int_{0}^{t} \int_{\Omega} \frac{A: \nabla u}{1-\frac{T r A}{b}}+\frac{1}{2}\left\|u_{0}\right\|_{L^{2}} .
\end{aligned}
$$

On the other hand, using 3.2 and the equation on the structure tensor $A$, we have

$$
\begin{aligned}
-\left(\partial_{t}+u \cdot \nabla\right) \ln (\operatorname{det} A) & =\frac{1}{W e} \frac{d}{1-\frac{\operatorname{Tr} A}{b}}-\frac{1}{W e} \operatorname{Tr}\left(A^{-1}\right), \\
-b\left(\partial_{t}+u \cdot \nabla\right) \ln \left(1-\frac{\operatorname{Tr} A}{b}\right) & =\frac{2 D(u): A}{1-\frac{\operatorname{Tr} A}{b}}-\frac{1}{W e} \frac{\operatorname{Tr} A}{\left(1-\frac{\operatorname{Tr} A}{b}\right)^{2}}+\frac{1}{W e} \frac{d}{1-\frac{\operatorname{Tr} A}{b}},
\end{aligned}
$$


which boils down to

$$
\begin{aligned}
\int_{\Omega}[-\ln (\operatorname{det} A)-b \ln & \left.\left(1-\frac{\operatorname{Tr} A}{b}\right)+(b+d) \ln \left(\frac{b}{b+d}\right)\right](T)=\int_{0}^{t} \int_{\Omega} \frac{2 D(u): A}{1-\frac{\operatorname{TrA}}{b}} \\
- & \frac{1}{W e} \int_{0}^{t} \int_{\Omega}\left[\frac{\operatorname{Tr} A}{\left(1-\frac{\operatorname{Tr} A}{b}\right)^{2}}-\frac{2 d}{1-\frac{\operatorname{TrA}}{b}}+\operatorname{Tr}\left(A^{-1}\right)\right] \\
& +\int_{\Omega}\left[-\ln \left(\operatorname{det} A_{0}\right)-b \ln \left(1-\frac{\operatorname{Tr} A_{0}}{b}\right)+(b+d) \ln \left(\frac{b}{b+d}\right)\right](t)
\end{aligned}
$$

The estimate 3.1$)$ is thus seen to hold thanks to the linear combination $(3.3)+\frac{1}{W e} \frac{(b+d) \omega}{2 b}(3.4)$. This proof actually serves as a model for our more complicated computations of the relative entropy estimates below.

As for the asymptotic analysis of the corotational system, we first investigate the weak convergence of $u$. In a second part, introducing corrector terms, we manage to prove the strong convergence of $u, A$ and $\tau$. The strong convergence is the truly tricky point.

3.1. Weak convergence. Letting $W e$ go to 0 in 1.2 yields formally that $A$ converges toward

$$
A^{0}:=\frac{b}{b+d} I \text {. }
$$

In the rest of this section we handle the proof of Proposition 1.2 .

The convergence of $A$ toward $A^{0}$ comes directly from the entropy inequality (3.1). The main observations, which lead to such a convergence result, are that the functionals

$$
\begin{aligned}
\mathcal{F} & : A \longmapsto-\ln (\operatorname{det} A)-b \ln \left(1-\frac{\operatorname{Tr} A}{b}\right)+(b+d) \ln \left(\frac{b}{b+d}\right) \\
\mathcal{H} & : A \longmapsto \frac{\operatorname{Tr} A}{\left(1-\frac{\operatorname{Tr} A}{b}\right)^{2}}-\frac{2 d}{1-\frac{\operatorname{Tr} A}{b}}+\operatorname{Tr}\left(A^{-1}\right)
\end{aligned}
$$

defined for appropriate symmetric positive definite matrices $A$ with $0<\operatorname{Tr} A<b$,

- have a global minimum at $A=A^{0}=\frac{b}{b+d} I$ with value 0 ,

- are globally strictly convex,

- and thus yield a bound on $\left|A-A^{0}\right|^{2}$.

Hence, one can use the decay of the free energy to prove the estimates 1.8, $1.10 \mathrm{a}$ and $1.10 \mathrm{~b}$. Notice that we also have the inequality $0 \leq \mathcal{F} \leq \mathcal{H}$.

We conclude, using these properties and the decay of the free energy that for all $t>0$,

$$
\begin{aligned}
0 \leq & \int_{\Omega}\left|A-A^{0}\right|^{2}(t) \leq C \int_{\Omega}\left[-\ln (\operatorname{det} A)-b \ln \left(1-\frac{\operatorname{Tr}(A)}{b}\right)+(b+d) \ln \left(\frac{b}{b+d}\right)\right](t) \\
\leq & C W e\left\|u_{0}\right\|_{L^{2}(\Omega)}^{2} \\
& \quad+\frac{\omega(b+d)}{2 b} \int_{\Omega}\left[-\ln \left(\operatorname{det} A_{0}\right)-b \ln \left(1-\frac{\operatorname{Tr}\left(A_{0}\right)}{b}\right)+(b+d) \ln \left(\frac{b}{b+d}\right)\right] .
\end{aligned}
$$

and that

$$
\begin{aligned}
0 & \leq \int_{0}^{t} \int_{\Omega}\left|A-A^{0}\right|^{2} \\
\leq & C \int_{0}^{t} \int_{\Omega}\left[\frac{\operatorname{Tr} A}{\left(1-\frac{\operatorname{TrA}}{b}\right)^{2}}-\frac{2 d}{1-\frac{\operatorname{TrA}}{b}}+\operatorname{Tr}\left(A^{-1}\right)\right] \\
\leq & \frac{1}{2} W e^{2}\left\|u_{0}\right\|_{L^{2}(\Omega)}^{2} \\
& \quad+\frac{\omega(b+2)}{2 b} W e \int_{\Omega}\left[-\ln \left(\operatorname{det} A_{0}\right)-b \ln \left(1-\frac{\operatorname{Tr}\left(A_{0}\right)}{b}\right)+(b+d) \ln \left(\frac{b}{b+d}\right)\right] .
\end{aligned}
$$


The bounds (3.7) and (3.8) imply the estimates of Proposition 1.2

It remains to establish the convergence of $u$ and $\tau$. Assume now that initial data is wellprepared, i.e. that 1.9$)$ is satisfied. We deduce a uniform $L^{2}\left((0, \infty) ; L^{2}\right)$ bound on $\tau$ from the convergence of $A$. Indeed, let us rewrite $\tau$ in the following way:

$$
\tau=\frac{(b+d) \omega}{b} \frac{1}{W e}\left[\frac{A}{1-\frac{\operatorname{TrA}}{b}}-I\right]=\frac{(b+d) \omega}{b} \frac{1}{W e}\left[\frac{A-A^{0}}{1-\frac{\operatorname{Tr} A^{0}}{b}}+A \frac{\operatorname{Tr} A-\operatorname{Tr} A^{0}}{b\left(1-\frac{\operatorname{Tr} A^{0}}{b}\right)\left(1-\frac{\operatorname{Tr} A}{b}\right)}\right] .
$$

The first term in the right hand side of 3.9

$$
\frac{1}{W e} \frac{A-A^{0}}{1-\frac{T r A^{0}}{b}}
$$

is bounded in $L^{2}\left((0, \infty) ; L^{2}\right)$ uniformly in $W e$, thanks to the convergence result $1.10 \mathrm{~b}$ ) for wellprepared data. For the second term, we notice that $A$ is bounded in $L^{\infty}\left((0, \infty) ; L^{\infty}\right)$ by $b$ and that

$$
\frac{(b+d) \omega}{b} \frac{1}{W e} \frac{\operatorname{Tr} A-\operatorname{Tr} A^{0}}{\left(1-\frac{\operatorname{Tr} A^{0}}{b}\right)\left(1-\frac{\operatorname{Tr} A}{b}\right)}=\frac{(b+d) \omega}{b} \frac{1}{W e}\left[\frac{\operatorname{Tr} A}{1-\frac{\operatorname{Tr} A}{b}}-\frac{\operatorname{Tr} A^{0}}{1-\frac{\operatorname{Tr} A^{0}}{b}}\right]=\operatorname{Tr} \tau .
$$

This part is bounded thanks to the decay of the free energy. Indeed, using the inequality

$$
\operatorname{Tr} A \operatorname{Tr}\left(A^{-1}\right) \geq d^{2}
$$

valid for the positive definite matrices $A$, we find that the fourth term in the right hand side of (3.1) bounds the $L^{2}\left((0, \infty) ; L^{2}\right)$ norm of $\operatorname{Tr} \tau$ :

$$
\begin{aligned}
\frac{\operatorname{Tr} A}{\left(1-\frac{\operatorname{Tr} A}{b}\right)^{2}}-\frac{2 d}{1-\frac{\operatorname{Tr} A}{b}}+\operatorname{Tr} A^{-1} & \geq \frac{\operatorname{Tr} A}{\left(1-\frac{\operatorname{Tr} A}{b}\right)^{2}}-\frac{2 d}{1-\frac{\operatorname{TrA}}{b}}+\frac{d^{2}}{\operatorname{Tr} A} \\
& \geq \frac{\left[\operatorname{Tr} A-d\left(1-\frac{\operatorname{Tr} A}{b}\right)\right]^{2}}{\operatorname{Tr} A\left(1-\frac{\operatorname{Tr} A}{b}\right)^{2}} \\
& \geq \frac{\left[\operatorname{Tr} A-d\left(1-\frac{\operatorname{Tr} A}{b}\right)\right]^{2}}{b\left(1-\frac{\operatorname{Tr} A}{b}\right)^{2}} \\
& =\frac{1}{b}\left[\frac{\operatorname{Tr} A}{1-\frac{\operatorname{Tr} A}{b}}-d\right]^{2} \geq \frac{b}{(b+d)^{2} \omega^{2}} W e^{2}(\operatorname{Tr} \tau)^{2} .
\end{aligned}
$$

It remains to see the convergence of $\tau$ and $u$. Assume that $d=2,3$. From (3.1), it comes that $u$ is uniformly bounded in $W e$ in $L^{\infty}\left((0, \infty) ; L^{2}\right)$ and $L^{2}\left((0, \infty) ; \dot{H}^{1}\right)$. Reasoning in the same manner as in Section 2.1, we deduce from the uniform bound on $\tau$ in $L^{2}((0, \infty) \times \Omega)$ the existence of

$$
u^{0} \in L^{\infty}\left((0, \infty) ; L^{2, \sigma}\right) \cap L^{2}\left((0, \infty) ; \dot{H}^{1}\right),
$$

such that $u$ converges to $u^{0}$ in a fashion similar to 2.1). To see the weak convergence of $\tau$, the idea is to use the equation on $A$ in the system 1.2 :

$$
\tau=-\frac{(b+d) \omega}{b}\left[\partial_{t} A+\nabla \cdot(u A)-\nabla u A-A(\nabla u)^{T}\right] .
$$

The right hand side of (3.11) converges in the sense of distributions toward

$$
-\frac{(b+d) \omega}{b}\left[\partial_{t} A^{0}+u^{0} \cdot \nabla A^{0}-2 \frac{b}{b+d} D\left(u^{0}\right)\right]=2 \omega D\left(u^{0}\right) .
$$

Finally, we can pass to the weak limit in the equation for $u^{0}$, and get that $u^{0}$ solves the NavierStokes system 1.6.

Remark 2. Notice that if one further assumes (1.9) with o(We) instead of $O(W e)$, we can prove that $u^{0}$ satisfies the energy estimate associated to the Navier-Stokes system. 
3.2. Strong convergence. This section is devoted to the proof of our main convergence result, namely Theorem 1.4. We aim at showing that $u$ converges strongly toward $u^{0}$ solving the Navier-Stokes system, for sufficiently nice initial data. Let

$$
u^{0} \in L^{\infty}\left((0, \infty) ; L^{2, \sigma}\right) \cap L^{2}\left((0, \infty) ; \dot{H}^{1}\right)
$$

be a global weak solution of the Navier-Stokes system 1.6, with no-slip boundary condition, associated to the regular initial data $u_{0} \in H^{4, \sigma}(\Omega)$. As in Section 2.2.2 there exists $0<T^{*} \leq \infty$ independent of $W e$ such that $u^{0}$, in addition, belongs to $L^{\infty}\left((0, T) ; H^{4}\right)$ for all $0<T<T^{*}$.

We follow the same steps as in the proof of the strong convergence for the corotational model (cf. Section 2.2):

1. Thanks to formal computations we identify relevant corrector terms for $u, A$ and $\tau$.

2. From the entropy for the FENE-P system, we then derive a formula for the relative entropy.

3. We finally show a Gronwall type estimate on the relative entropy.

This scheme proves more complicated due to the complex form of the free energy (3.1) associated to the FENE-P system in comparison to the simple energy estimate (1.3). From the analysis carried out in Section 3.1, we already know that we have the following control

$$
\begin{aligned}
& \frac{\omega(b+d)}{2 b} \frac{1}{W e}\left\|A(t, \cdot)-A^{0}(t, \cdot)\right\|_{L^{2}}^{2}+\frac{\omega(b+d)}{2 b} \frac{1}{W e^{2}} \int_{0}^{t}\left\|A-A^{0}\right\|_{L^{2}}^{2} \\
& \leq \frac{\omega(b+d)}{2 b} \frac{1}{W e} \int_{\Omega}\left[-\ln (\operatorname{det} A)-b \ln \left(1-\frac{\operatorname{Tr}(A)}{b}\right)+(b+d) \ln \left(\frac{b}{b+d}\right)\right](t) \\
& \quad+\frac{\omega(b+d)}{2 b} \frac{1}{W e^{2}} \int_{0}^{t} \int_{\Omega}\left[\frac{\operatorname{Tr} A}{\left(1-\frac{\operatorname{Tr} A}{b}\right)^{2}}-\frac{2 d}{1-\frac{\operatorname{Tr} A}{b}}+\operatorname{Tr}\left(A^{-1}\right)\right] .
\end{aligned}
$$

However, as emphasized in the proof of the strong convergence for the corotational model, we need to push the expansions of $u, A$ and $\tau$ up to the order 1 in $W e$, in order end up at a Gronwall inequality with a small source term, of order $O(W e)$. This requires to handle the term

$$
-\left(\tau-\tau^{0}\right): \nabla\left(u-u^{0}-W e u^{1}\right)
$$

coming from a weak strong energy estimate on the momentum equation. One of our main difficulties is that for the FENE-P system the transport equation holds on the structure tensor $A$, not on the stress tensor $\tau$ as in the corotational system (1.1), and that $A$ and $\tau$ are related by a non-trivial nonlinear relation. This accounts for the difficulty to extract the terms in 3.12 from our equations.

We face this issue by expanding the entropy (3.1) around the corrector $A^{0}+W e A^{1}$. We thus derive a new entropy, the relative entropy (3.17), for the FENE-P system, relevant to the study of the low Weissenberg asymptotics. Notice that expanding the entropy around $A^{0}$ rather than around $A^{0}+W e A^{1}$ would not be enough.

The expansion of the relative entropy in terms of $W e$ makes it possible to underline the correspondence between the corotational and the FENE-P systems. Roughly speaking, our leading idea is that the terms at main order $O(1)$ in $W e$ can be handled similarly in both cases. The other terms are remainder terms, which are shown to be small and hence do not make a difference at the limit.

3.2.1. Formal computation of the corrector terms. We simply rewrite the FENE-P system in terms of $\Pi(A):=\frac{A}{1-\frac{T r A}{b}}-I$ :

$$
\left\{\begin{array}{rl}
\partial_{t} u+u \cdot \nabla u-(1-\omega) \Delta u+\nabla p & =\frac{(b+d) \omega}{b} \frac{1}{W e} \nabla \cdot \Pi(A) \\
\nabla \cdot u & =0 \\
\partial_{t} A+u \cdot \nabla A-\nabla u A-A(\nabla u)^{T}+\frac{1}{W e} \Pi(A) & =0 \\
\tau & =\frac{(b+d) \omega}{b} \frac{1}{W e} \Pi(A)
\end{array} .\right.
$$

As for the corotational system, we begin with the formal computation of the corrector terms. Let us assume that

$$
u \simeq u^{0}+W e u^{1}, \quad p \simeq p^{0}+W e p^{1}, \quad A \simeq A^{0}+W e A^{1}+W e^{2} A^{2} .
$$


Expanding $\Pi$ near $A^{0}$ yields:

$$
\Pi\left(A^{0}+H\right)=\Pi\left(A^{0}\right)+(D \Pi)\left(A^{0}\right) H+\frac{1}{2}\left(D^{2} \Pi\right)\left(A^{0}\right)(H, H)+O\left(H^{3}\right) .
$$

Hence, one infers

$$
\begin{aligned}
& \Pi\left(A^{0}+W e A^{1}+W e^{2} A^{2}\right)=\frac{A^{0}}{1-\frac{\operatorname{Tr} A^{0}}{b}}-I \\
& +W e\left[\frac{A^{0} \operatorname{Tr} A^{1}}{b\left(1-\frac{\operatorname{Tr} A^{0}}{b}\right)^{2}}+\frac{A^{1}}{1-\frac{\operatorname{Tr} A^{0}}{b}}\right] \\
& +W e^{2}\left[\frac{A^{0} \operatorname{Tr} A^{2}}{b\left(1-\frac{\operatorname{Tr} A^{0}}{b}\right)^{2}}+\frac{A^{2}}{1-\frac{\operatorname{Tr} A^{0}}{b}}+\frac{A^{0}\left(\operatorname{Tr} A^{1}\right)^{2}}{2 b^{2}\left(1-\frac{\operatorname{Tr} A^{0}}{b}\right)^{3}}+\frac{A^{1} \operatorname{Tr} A^{1}}{2 b\left(1-\frac{\operatorname{Tr} A^{0}}{b}\right)^{2}}\right] \\
& +O\left(W e^{3}\right) .
\end{aligned}
$$

Plugging the ansatz in the momentum equation, we get that $u^{0}$ solves the Navier-Stokes system (1.6), and at first order in $W e$ that $u^{1}$ solves

$$
\begin{aligned}
\partial_{t} u^{1}+u^{0} & \nabla u^{1}+u^{1} \cdot \nabla u^{0}-(1-\omega) \Delta u^{1}+\nabla p^{1} \\
& =\frac{(b+d) \omega}{b} \nabla \cdot\left[\frac{A^{0} \operatorname{Tr} A^{2}}{b\left(1-\frac{T r A^{0}}{b}\right)^{2}}+\frac{A^{2}}{1-\frac{T r A^{0}}{b}}+\frac{A^{0}\left(\operatorname{Tr} A^{1}\right)^{2}}{2 b^{2}\left(1-\frac{\operatorname{Tr} A^{0}}{b}\right)^{3}}+\frac{A^{1} \operatorname{Tr} A^{1}}{2 b\left(1-\frac{\operatorname{Tr} A^{0}}{b}\right)^{2}}\right] .
\end{aligned}
$$

Note that both $u^{0}$ and $u^{1}$ are incompressible fields, and meet the no-slip boundary condition on $\partial \Omega$. We impose the initial condition $u^{1}(0, \cdot)=0$.

Plugging now the ansatz in the equation on the structure tensor $A$ and identifying the orders in $W e$ leads to

$$
\begin{aligned}
& \frac{A^{0}}{1-\frac{\operatorname{Tr} A^{0}}{b}}-I=0, \\
& \partial_{t} A^{0}+u^{0} \cdot \nabla A^{0}-\nabla u^{0} A^{0}-A^{0}\left(\nabla u^{0}\right)^{T}+\frac{A^{0} \operatorname{Tr} A^{1}}{b\left(1-\frac{\operatorname{Tr} A^{0}}{b}\right)^{2}}+\frac{A^{1}}{1-\frac{\operatorname{Tr} A^{0}}{b}}=0, \\
& \partial_{t} A^{1}+u^{0} \cdot \nabla A^{1}+u^{1} \cdot \nabla A^{0}-\nabla u^{1} A^{0}-\nabla u^{0} A^{1}-A^{1}\left(\nabla u^{0}\right)^{T}-A^{0}\left(\nabla u^{1}\right)^{T} \\
& \quad+\frac{A^{0} \operatorname{Tr} A^{2}}{b\left(1-\frac{\operatorname{Tr} A^{0}}{b}\right)^{2}}+\frac{A^{2}}{1-\frac{\operatorname{Tr} A^{0}}{b}}+\frac{A^{0}\left(\operatorname{Tr} A^{1}\right)^{2}}{2 b^{2}\left(1-\frac{\operatorname{Tr} A^{0}}{b}\right)^{3}}+\frac{A^{1} \operatorname{Tr} A^{1}}{2 b\left(1-\frac{\operatorname{Tr} A^{0}}{b}\right)^{2}}=0,
\end{aligned}
$$

from which we retrieve

$$
\begin{aligned}
A^{0} & =\frac{b}{b+d} I \\
A^{1} & =2\left(\frac{b}{b+d}\right)^{2} D\left(u^{0}\right) \text { and } \operatorname{Tr} A^{1}=0, \\
\operatorname{Tr} A^{2} & =4\left(\frac{b}{b+d}\right)^{4} D\left(u^{0}\right): D\left(u^{0}\right) .
\end{aligned}
$$

Letting

$$
\tau^{1}:=\frac{(b+d) \omega}{b}\left[\frac{A^{0} \operatorname{Tr} A^{2}}{b\left(1-\frac{\operatorname{Tr} A^{0}}{b}\right)^{2}}+\frac{A^{2}}{1-\frac{\operatorname{Tr} A^{0}}{b}}\right]
$$

we notice that

$$
\partial_{t} A^{1}+u^{0} \cdot \nabla A^{1}-\nabla u^{1} A^{0}-A^{0}\left(\nabla u^{1}\right)^{T}-\nabla u^{0} A^{1}-A^{1}\left(\nabla u^{0}\right)^{T}+\frac{b}{\omega(b+d)} \tau^{1}=0 .
$$


REMARK 3 (Analogy with the corotational system). Again, there are some similarities between the correctors $\tau^{0}$ for the corotational system, and $A^{1}$ for the FENE-P system. The equation (2.4) for $\tau^{0}$ and the equation (3.15) have an analogous structure, and $A^{1}$ and $\tau^{0}$ are equal up to a constant. This parallel is a leitmotiv of our further computations.

REMARK 4 (On the regularity of the corrector terms). Let us say some words about the regularity of the profiles. We argue exactly as in Section 2.2.2. As soon as $u_{0} \in H^{4}(\Omega)$, we get that

$$
\begin{aligned}
& u^{0} \in L^{\infty}\left((0, T) ; H^{4}\right), \\
& u^{1} \in L^{\infty}\left((0, T) ; H^{1}\right), \\
& A^{1} \in L^{\infty}\left((0, T) ; H^{3}\right) \subset L^{\infty}((0, T) \times \Omega), \\
& \tau^{0} \in L^{\infty}\left((0, T) ; H^{3}\right), \\
& \tau^{1} \in L^{2}\left((0, T) ; L^{2}\right) .
\end{aligned}
$$

REMARK 5 (On the definite positivity of the correctors). The first-order corrector $A^{0}$ is evidently definite positive. For We sufficiently small, this happens to be also the case for $A^{0}+$ $W e A^{1}$, uniformly in $t$ and $x$. This follows from the fact that $D\left(u^{0}\right)$ belongs to $L^{\infty}((0, T) \times \Omega)$.

3.2.2. Relative entropy: expansion of the free energy at first-order.

Expansion of $\mathcal{F}$. Let us consider

$$
\widetilde{\mathcal{F}}: A \longmapsto \mathcal{F}(A)-\mathcal{F}\left(A^{0}+W e A^{1}\right)-D \mathcal{F}\left(A^{0}+W e A^{1}\right)\left(A-A^{0}-W e A^{1}\right),
$$

where $\mathcal{F}$ is defined by $(3.5)$. Expanding $\mathcal{F}$ by the Taylor formula yields

$$
\begin{aligned}
-\ln (\operatorname{det}(B+H)) & =-\ln (\operatorname{det}(B))-\operatorname{Tr}\left(B^{-1} H\right)+O\left(H^{2}\right), \\
-b \ln \left(1-\frac{\operatorname{Tr}(B+H)}{b}\right) & =-b \ln \left(1-\frac{\operatorname{Tr} B}{b}\right)+\frac{\operatorname{Tr} H}{1-\frac{\operatorname{Tr} B}{b}}+O\left(H^{2}\right), \\
\mathcal{F}(B+H) & =\mathcal{F}(B)-\operatorname{Tr}\left(B^{-1} H\right)+\frac{\operatorname{Tr} H}{1-\frac{\operatorname{Tr} B}{b}}+O\left(H^{2}\right) .
\end{aligned}
$$

Therefore,

$$
\begin{aligned}
\widetilde{\mathcal{F}}(A)=-\ln (\operatorname{det}(A))- & \ln \left(1-\frac{\operatorname{Tr} A}{b}\right)+\ln \left(\operatorname{det}\left(A^{0}+W e A^{1}\right)\right)+b \ln \left(1-\frac{\operatorname{Tr}\left(A^{0}+W e A^{1}\right)}{b}\right) \\
+ & \operatorname{Tr}\left(\left(A^{0}+W e A^{1}\right)^{-1}\left(A-A^{0}-W e A^{1}\right)\right)-\frac{\operatorname{Tr}\left(A-A^{0}-W e A^{1}\right)}{1-\frac{\operatorname{Tr} A^{0}}{b}} .
\end{aligned}
$$

The positivity of the Hessian matrix and the Taylor formula with integral rest at order 1

$$
\begin{aligned}
\mathcal{F}(A)=\mathcal{F}\left(A^{0}+\right. & \left.W e A^{1}\right)+D \mathcal{F}\left(A^{0}+W e A^{1}\right)\left(A-A^{0}-W e A^{1}\right) \\
& +\int_{0}^{1}(1-t) D^{2} \mathcal{F}\left(A^{0}+W e A^{1}+t\left(A-A^{0}-W e A^{1}\right)\right)\left(A-A^{0}-W e A^{1}\right)^{2} d t
\end{aligned}
$$

shows that $\tilde{\mathcal{F}}(A)$ is positive and bounds $\left|A-A^{0}-W e A^{1}\right|^{2}$.

Expansion of $\mathcal{H}$. In the same fashion, we consider

$$
\widetilde{\mathcal{H}}: A \longmapsto \mathcal{H}(A)-\mathcal{H}\left(A^{0}+W e A^{1}\right)-D \mathcal{H}\left(A^{0}-W e A^{1}\right)\left(A-A^{0}-W e A^{1}\right) .
$$

where $\mathcal{H}$ is defined by $(3.6)$. Expanding $\mathcal{H}$ by the Taylor formula yields

$$
\begin{aligned}
& \frac{\operatorname{Tr}(B+H)}{\left(1-\frac{\operatorname{Tr}(B+H)}{b}\right)^{2}}=\frac{\operatorname{Tr} B}{\left(1-\frac{\operatorname{Tr} B}{b}\right)^{2}}+\frac{1}{\left(1-\frac{\operatorname{Tr} B}{b}\right)^{2}}\left[1+\frac{2 \operatorname{Tr} B}{b\left(1-\frac{\operatorname{Tr} B}{b}\right)}\right] \operatorname{Tr} H+O\left(H^{2}\right), \\
& -\frac{2 d}{1-\frac{\operatorname{Tr}(B+H)}{b}}=-\frac{2 d}{1-\frac{\operatorname{Tr} B}{b}}-\frac{2 d \operatorname{Tr} H}{b\left(1-\frac{\operatorname{Tr} B}{b}\right)^{2}}+O\left(H^{2}\right), \\
& \operatorname{Tr}\left((B+H)^{-1}\right)=\operatorname{Tr}\left(B^{-1}\right)-\operatorname{Tr}\left(\frac{H}{B^{2}}\right)+O\left(H^{2}\right),
\end{aligned}
$$


so that

$$
\begin{aligned}
& \widetilde{\mathcal{H}}(A)=\frac{\operatorname{Tr} A}{\left(1-\frac{\operatorname{Tr} A}{b}\right)^{2}}-\frac{2 d}{1-\frac{\operatorname{Tr} A}{b}}+\operatorname{Tr}\left(A^{-1}\right) \\
& -\frac{\operatorname{Tr}\left(A^{0}+W e A^{1}\right)}{\left(1-\frac{\operatorname{Tr}\left(A^{0}+W e A^{1}\right)}{b}\right)^{2}}+\frac{2 d}{1-\frac{\operatorname{Tr}\left(A^{0}+W e A^{1}\right)}{b}}-\operatorname{Tr}\left(\left(A^{0}+W e A^{1}\right)^{-1}\right) \\
& -\frac{1}{\left(1-\frac{\operatorname{Tr}\left(A^{0}+W e A^{1}\right)}{b}\right)^{2}}\left[1+\frac{2 \operatorname{Tr}\left(A^{0}+W e A^{1}\right)}{b\left(1-\frac{\operatorname{Tr}\left(A^{0}+W e A^{1}\right)}{b}\right)}\right] \operatorname{Tr}\left(A-A^{0}-W e A^{1}\right) \\
& +\frac{2 d \operatorname{Tr}\left(A-A^{0}-W e A^{1}\right)}{b\left(1-\frac{\operatorname{Tr}\left(A^{0}+W e A^{1}\right)}{b}\right)^{2}}+\operatorname{Tr}\left(\frac{A-A^{0}-W e A^{1}}{\left(A^{0}+W e A^{1}\right)^{2}}\right) \\
& =\frac{\operatorname{Tr} A}{\left(1-\frac{\operatorname{Tr} A}{b}\right)^{2}}-\frac{2 d}{1-\frac{\operatorname{Tr} A}{b}}+\operatorname{Tr}\left(A^{-1}\right) \\
& -\frac{\operatorname{Tr} A^{0}}{\left(1-\frac{\operatorname{Tr} A^{0}}{b}\right)^{2}}+\frac{2 d}{1-\frac{\operatorname{Tr} A^{0}}{b}}-\operatorname{Tr}\left(\left(A^{0}+W e A^{1}\right)^{-1}\right) \\
& -\frac{\operatorname{Tr}\left(A-A^{0}-W e A^{1}\right)}{\left(1-\frac{\operatorname{Tr} A^{0}}{b}\right)^{2}}+\operatorname{Tr}\left(\frac{A-A^{0}-W e A^{1}}{\left(A^{0}+W e A^{1}\right)^{2}}\right) \text {. }
\end{aligned}
$$

The positivity of the Hessian matrix and the Taylor formula with integral rest at order 1

$$
\begin{aligned}
\mathcal{H}(A)=\mathcal{H}\left(A^{0}+\right. & \left.W e A^{1}\right)+D \mathcal{H}\left(A^{0}+W e A^{1}\right)\left(A-A^{0}-W e A^{1}\right) \\
& +\int_{0}^{1}(1-t) D^{2} \mathcal{H}\left(A^{0}+W e A^{1}+t\left(A-A^{0}-W e A^{1}\right)\right)\left(A-A^{0}-W e A^{1}\right)^{2} d t
\end{aligned}
$$

shows that $\widetilde{\mathcal{H}}(A)$ is positive and bounds $\left|A-A^{0}-W e A^{1}\right|^{2}$.

These remarks lead us to consider the quantity

$$
\begin{aligned}
\mathfrak{E}(t):=\frac{1}{2}\left\|U^{(r)}(t, \cdot)\right\|_{L^{2}}^{2}+(1 & -\omega) \int_{0}^{t}\left\|\nabla U^{(r)}\right\|_{L^{2}}^{2} \\
& +\frac{\omega(b+d)}{2 b} \frac{1}{W e} \int_{\Omega} \widetilde{\mathcal{F}}(A)(t, \cdot)+\frac{\omega(b+d)}{2 b} \frac{1}{W e^{2}} \int_{0}^{t} \int_{\Omega} \widetilde{\mathcal{H}}(A) .
\end{aligned}
$$

We call it the relative entropy of our system. Its role is analogous to the relative entropy (2.17) for the corotational system.

3.2.3. Estimate of the relative entropy. To put it in a nutshell, our purpose is to establish a Gronwall type inequality on

$$
\begin{aligned}
\mathfrak{D}:=\frac{1}{2}\left\|U^{(r)}(t, \cdot)\right\|_{L^{2}}^{2}+(1-\omega) \int_{0}^{t}\left\|\nabla U^{(r)}\right\|_{L^{2}}^{2} & \\
& +\frac{\omega(b+d)}{2 b} \frac{1}{W e}\left\|A-A^{0}-W e A^{1}\right\|_{L^{2}}^{2}+\frac{\omega(b+d)}{2 b} \frac{1}{W e^{2}} \int_{0}^{t}\left\|A-A^{0}-W e A^{1}\right\|_{L^{2}}^{2},
\end{aligned}
$$

for $d=2,3$. This latter quantity is, of course, smaller than the relative entropy: $\mathfrak{D} \leq C \mathfrak{E}$. Roughly speaking, the Gronwall inequality should look like

$$
\mathfrak{D} \leq \mathfrak{E}(0)+\int_{0}^{t} \mathcal{C}(s) \mathfrak{D}(s) d s+O(W e),
$$

where $s \mapsto \mathcal{C}(s)$ is a positive and locally bounded function. Notice that this is exactly what we did for the corotational system: see in particular 2.16). If we manage to prove (3.18), we can expect 
to get the following bounds:

$$
\begin{aligned}
\left\|u-u^{0}\right\|_{L^{2}\left((0, T) ; H^{1}\right)} & =O(\sqrt{W e}), \\
\left\|A-A^{0}\right\|_{L^{\infty}\left((0, T) ; L^{2}\right)} & =O(W e), \\
\left\|A-A^{0}-W e A^{1}\right\|_{L^{2}\left((0, T) ; L^{2}\right)} & =O\left(W e^{3 / 2}\right),
\end{aligned}
$$

provided that $\mathfrak{E}(0)=O(W e)$ (well-prepared initial data for $u$ and $A$ ). Moreover, improving the bound on $A-A^{0}-W e A^{1}$ yields a better bound on $\tau-\tau^{0}$ in $L^{1}$. This is a consequence of the formulas

$$
\begin{aligned}
\tau-\tau^{0} & =\frac{(b+d) \omega}{b} \frac{1}{W e} \Pi(A)-2 \omega D\left(u^{0}\right) \\
& =\frac{(b+d) \omega}{b} \frac{1}{W e}\left[\frac{A-A^{0}-W e A^{1}}{1-\frac{\operatorname{Tr} A^{0}}{b}}+A \frac{\operatorname{Tr} A-\operatorname{Tr} A^{0}-W e \operatorname{Tr} A^{1}}{b\left(1-\frac{\operatorname{Tr} A^{0}}{b}\right)\left(1-\frac{\operatorname{Tr} A}{b}\right)}\right] .
\end{aligned}
$$

and

$$
\begin{aligned}
& \frac{1}{W e} A \frac{\operatorname{Tr} A-\operatorname{Tr} A^{0}-W e \operatorname{Tr} A^{1}}{b\left(1-\frac{\operatorname{Tr} A^{0}}{b}\right)\left(1-\frac{\operatorname{Tr} A}{b}\right)} \\
& =\frac{1}{W e}\left(\frac{A}{1-\frac{\operatorname{Tr} A}{b}}-I+I\right) \frac{\operatorname{Tr} A-\operatorname{Tr} A^{0}-W e \operatorname{Tr} A^{1}}{b\left(1-\frac{\operatorname{Tr} A^{0}}{b}\right)} \\
& =\frac{1}{W e}\left(\frac{A}{1-\frac{\operatorname{Tr} A}{b}}-I\right) \frac{\operatorname{Tr} A-\operatorname{Tr} A^{0}-W e \operatorname{Tr} A^{1}}{b\left(1-\frac{\operatorname{Tr} A^{0}}{b}\right)}+\frac{1}{W e} \frac{\operatorname{Tr} A-\operatorname{Tr} A^{0}-W e \operatorname{Tr} A^{1}}{b\left(1-\frac{\operatorname{Tr} A^{0}}{b}\right)} .
\end{aligned}
$$

In order to establish a Gronwall type estimate on $\mathfrak{D}$, we compute each of the terms appearing in (3.17). The two first terms can be handle thanks to an energy estimate on the momentum equation. We have

$$
\begin{aligned}
\partial_{t} u+u \cdot \nabla u-(1-\omega) \Delta u+\nabla p & =\nabla \cdot \tau, \\
\partial_{t} u^{0}+u \cdot \nabla u^{0}-(1-\omega) \Delta u^{0}+\nabla p^{0} & =\nabla \cdot \tau^{0}+\left(u-u^{0}\right) \cdot \nabla u^{0}, \\
\partial_{t} u^{1}+u \cdot \nabla u^{1}-(1-\omega) \Delta u^{1}+\nabla p^{1} & =\nabla \cdot \tau^{1}+\left(u-u^{0}\right) \cdot \nabla u^{1}-u^{1} \cdot \nabla u^{0},
\end{aligned}
$$

which yields

$$
\begin{aligned}
\left(\partial_{t}+u \cdot \nabla\right)(u- & \left.u^{0}-W e u^{1}\right)-(1-\omega) \Delta\left(u-u^{0}-W e u^{1}\right)+\nabla\left(p-p^{0}-W e p^{1}\right) \\
& =\nabla \cdot\left(\tau-\tau^{0}-W e \tau^{1}\right)-\left(u-u^{0}-W e u^{1}\right) \cdot \nabla u^{0}-W e\left(u-u^{0}\right) \cdot \nabla u^{1} .
\end{aligned}
$$

Testing the equation 3.23 against $U^{(r)}:=u-u^{0}-W e u^{1}$, yields the estimate of the following lemma.

LEMMA 3.1. We have the energy estimate

$$
\begin{aligned}
\frac{1}{2}\left\|U^{(r)}(t, \cdot)\right\|_{L^{2}}^{2} & +(1-\omega) \int_{0}^{t}\left\|\nabla U^{(r)}\right\|_{L^{2}}^{2} \\
=-\int_{0}^{t} \int_{\Omega}\left(\tau-\tau^{0}-W e \tau^{1}\right): \nabla U^{(r)} & -\int_{0}^{t} \int_{\Omega}\left(U^{(r)} \cdot \nabla u^{0}\right) \cdot U^{(r)} \\
& -W e \int_{0}^{t} \int_{\Omega}\left(\left(u-u^{0}\right) \cdot \nabla u^{1}\right) \cdot U^{(r)} .
\end{aligned}
$$

We split the right hand side of (3.24) into terms which are of order $O(W e)$

$$
\begin{aligned}
& -W e \int_{0}^{t} \int_{\Omega} \tau^{0}: \nabla u^{1}, \quad W e \int_{0}^{t} \int_{\Omega} \tau^{1}: \nabla U^{(r)}, \quad W e \int_{0}^{t} \int_{\Omega} \tau: \nabla u^{1}, \\
& -W e \int_{0}^{t} \int_{\Omega}\left(\left(u-u^{0}\right) \cdot \nabla u^{1}\right) \cdot U^{(r)}
\end{aligned}
$$


a term which allows to close the Gronwall estimate

$$
-\int_{0}^{t} \int_{\Omega}\left(U^{(r)} \cdot \nabla u^{0}\right) \cdot U^{(r)}
$$

and other terms

$$
-\int_{0}^{t} \int_{\Omega} \tau: \nabla u, \quad-\int_{0}^{t} \int_{\Omega} \tau^{0}: \nabla u^{0}, \quad \int_{0}^{t} \int_{\Omega} \tau: \nabla u^{0}, \quad \int_{0}^{t} \int_{\Omega} \tau^{0}: \nabla u,
$$

which need not be small. Luckily enough, we manage to show that the latter terms do not appear in the final estimate of the relative entropy.

We now state and prove three key lemmas showing that the terms of the relative entropy involving $\widetilde{\mathcal{F}}(A)$ and $\widetilde{\mathcal{H}}(A)$ cancel the terms in 3.25 . From the proof of the entropy estimate 3.1 , we already know that this holds for the term $-\int_{0}^{t} \int_{\Omega} \tau: \nabla u$.

LEMMA 3.2. We have

$$
\begin{aligned}
\frac{\omega(b+d)}{2 b} \frac{1}{W e}\left[\int_{\Omega} \mathcal{F}(A)+\frac{1}{W e} \int_{0}^{t} \int_{\Omega} \mathcal{H}(A)\right] & \\
& =\left.\frac{\omega(b+d)}{2 b} \frac{1}{W e} \int_{\Omega} \mathcal{F}(A)\right|_{t=0}+\int_{0}^{t} \int_{\Omega} \tau: \nabla u .
\end{aligned}
$$

The next lemma indicates how to get rid of the term $-\int_{0}^{t} \int_{\Omega} \tau^{0}: \nabla u^{0}$ :

LEMMA 3.3. We have

$$
\begin{aligned}
-\frac{\omega(b+d)}{2 b} \frac{1}{W e}[ & \left.\int_{\Omega} \mathcal{F}\left(A^{0}+W e A^{1}\right)+\frac{1}{W e} \int_{0}^{t} \int_{\Omega} \mathcal{H}\left(A^{0}+W e A^{1}\right)\right] \\
& =-\left.\frac{\omega(b+d)}{2 b} \frac{1}{W e} \int_{\Omega} \mathcal{F}\left(A^{0}+W e A^{1}\right)\right|_{t=0}-\int_{0}^{t} \int_{\Omega} \tau^{0}: \nabla u^{0}+O(W e) .
\end{aligned}
$$

Let us notice that the sign of $-\int_{0}^{t} \int_{\Omega} \tau^{0}: \nabla u^{0}$ is precisely opposite to the sign we would like in order to kill $-\int_{0}^{t} \int_{\Omega} \tau^{0}: \nabla u^{0}$ (cf. 3.25 ). However, we will see (cf. Lemma 3.4), that the term

$$
-\frac{1}{W e^{2}} \frac{\omega(b+d)}{2 b} \int_{0}^{t} \int_{\Omega} D \mathcal{H}\left(A^{0}+W e A^{1}\right)\left(A-A^{0}-W e A^{1}\right) .
$$

adds the quantity $2 \int_{0}^{t} \int_{\Omega} \tau^{0}: \nabla u^{0}$, so that we will recover what we wish.

Proof. [Proof of Lemma 3.3] The proof consists merely in a computation of the total time derivative $\partial_{t}+u \cdot \nabla$ of the left hand side of $(3.27)$. Note that we often use the fact that $\operatorname{Tr}\left(\left(\partial_{t}+u \cdot \nabla\right) A^{1}\right)=0$. Expanding in powers of $W e$, we get

$$
\begin{aligned}
& -\left(\partial_{t}+u \cdot \nabla\right) \mathcal{F}\left(A^{0}+W e A^{1}\right)-\frac{1}{W e} \mathcal{H}\left(A^{0}+W e A^{1}\right) \\
& =W e \operatorname{Tr}\left(\left(A^{0}+W e A^{1}\right)^{-1}\left(\partial_{t}+u \cdot \nabla\right) A^{1}\right) \\
& -\frac{1}{W e}\left[\frac{\operatorname{Tr} A^{0}}{\left(1-\frac{\operatorname{Tr} A^{0}}{b}\right)^{2}}-\frac{2 d}{1-\frac{\operatorname{Tr} A^{0}}{b}}+\operatorname{Tr}\left(\left(A^{0}+W e A^{1}\right)^{-1}\right)\right] \\
& =-W e \operatorname{Tr}\left(\frac{\left(A^{1}\right)^{2}}{\left(A^{0}\right)^{3}}\right)+W e^{2}\left[\sum_{k=1}^{\infty}(-1)^{k} W e^{k-1} \operatorname{Tr}\left(\frac{\left(A^{1}\right)^{k}}{\left(A^{0}\right)^{k+1}}\left(\partial_{t}+u \cdot \nabla\right) A^{1}\right)\right. \\
& \left.-\sum_{k=3}^{\infty} W e^{k-3}(-1)^{k} \operatorname{Tr}\left(\frac{\left(A^{1}\right)^{k}}{\left(A^{0}\right)^{k+1}}\right)\right] .
\end{aligned}
$$

We focus on the term of order $W e$. It follows from the expression 3.14 of $A^{1}$ that

$$
-\frac{\omega(b+d)}{2 b} \int_{0}^{t} \int_{\Omega} \operatorname{Tr}\left(\frac{\left(A^{1}\right)^{2}}{\left(A^{0}\right)^{3}}\right)=-\tau^{0}: D\left(u^{0}\right) .
$$


Therefore (3.27) holds with a remainder term equal to

$$
\begin{aligned}
& \frac{\omega(b+d)}{2 b} W e \int_{0}^{t} \int_{\Omega}\left[\sum_{k=1}^{\infty}(-1)^{k} W e^{k-1} \operatorname{Tr}\left(\frac{\left(A^{1}\right)^{k}}{\left(A^{0}\right)^{k+1}}\left(\partial_{t}+u \cdot \nabla\right) A^{1}\right)\right. \\
& \left.\quad-\sum_{k=3}^{\infty} W e^{k-3}(-1)^{k} \operatorname{Tr}\left(\frac{\left(A^{1}\right)^{k}}{\left(A^{0}\right)^{k+1}}\right)\right]=O(W e),
\end{aligned}
$$

which concludes the proof of the lemma.

REMARK 6 (On the remainder term). The notation $O(W e)$ in (3.27) hides the structure of the remainder term. The term

$$
\frac{\omega(b+d)}{2 b} W e \int_{0}^{t} \int_{\Omega} \sum_{k=1}^{\infty}(-1)^{k} W e^{k-1} \operatorname{Tr}\left(\frac{\left(A^{1}\right)^{k}}{\left(A^{0}\right)^{k+1}}\left(\partial_{t}+u \cdot \nabla\right) A^{1}\right)
$$

in (3.28) involves $u$. One can rely on the standard splitting of the total time derivative

$$
\partial_{t}+u \cdot \nabla=\partial_{t}+u^{0} \cdot \nabla+\left(u-u^{0}-W e u^{1}\right) \cdot \nabla+W e u^{1} \cdot \nabla,
$$

and estimate the term

$$
\begin{aligned}
& \left|\frac{\omega(b+d)}{2 b} W e \int_{0}^{t} \int_{\Omega} \sum_{k=1}^{\infty}(-1)^{k} W e^{k-1} \operatorname{Tr}\left(\frac{\left(A^{1}\right)^{k}}{\left(A^{0}\right)^{k+1}}\left(u-u^{0}-W e u^{1}\right) \cdot \nabla A^{1}\right)\right| \\
& \leq \frac{\omega(b+d)}{4 b} W e\left[\sum_{k=1}^{\infty} W e^{k-1} \int_{0}^{t} \int_{\Omega}\left|\frac{\left(A^{1}\right)^{k}}{\left(A^{0}\right)^{k+1}}\right|^{2}+\sum_{k=1}^{\infty} W e^{k-1} \int_{0}^{t} \int_{\Omega}\left|\left(u-u^{0}-W e u^{1}\right) \cdot \nabla A^{1}\right|^{2}\right] .
\end{aligned}
$$

Then the first term in the right hand side above is $O(W e)$, and the second term can be absorbed in $\int_{0}^{t} \mathcal{C}(s) \mathfrak{D}(s) d s$ (see the Gronwall inequality (3.18)).

REMARK 7 (On the convergence of the infinite series). Using the regularity statements (3.16) and the identity 3.29 , we notice that

$$
\left(\partial_{t}+u \cdot \nabla\right) A^{1} \in L^{2}((0, T) \times \Omega)
$$

so that for all $k \geq 1$,

$$
\begin{aligned}
& \int_{0}^{t} \int_{\Omega}\left|(-1)^{k} W e^{k-1} \operatorname{Tr}\left(\frac{\left(A^{1}\right)^{k}}{\left(A^{0}\right)^{k+1}}\left(\partial_{t}+u \cdot \nabla\right) A^{1}\right)\right| \\
& \leq W e^{k-1}\left(\frac{b+d}{b}\right)^{k+1}\left(\int_{0}^{t} \int_{\Omega}\left|A^{1}\right|^{2 k}\right)^{\frac{1}{2}}\left\|\left(\partial_{t}+u \cdot \nabla\right) A^{1}\right\|_{L^{2}((0, T) \times \Omega)} \\
& \leq W e^{k-1}\left(\frac{b+d}{b}\right)^{k+1}\left\|A^{1}\right\|_{L^{\infty}\left((0, T) ; H^{3}\right)}^{k}\left\|\left(\partial_{t}+u \cdot \nabla\right) A^{1}\right\|_{L^{2}((0, T) \times \Omega)} .
\end{aligned}
$$

Thus, the series converges for We sufficiently small, i.e.

$$
0<W e<\frac{b}{b+d} \frac{1}{\left\|A^{1}\right\|_{L^{\infty}\left((0, T) ; H^{3}\right)}} .
$$

We finally show that the crossed terms $\int_{0}^{t} \int_{\Omega} \tau: \nabla u^{0}$ and $\int_{0}^{t} \int_{\Omega} \tau^{0}: \nabla u$ can be recovered thanks to the first-order terms in the Taylor expansion of $\mathcal{F}$ and $\mathcal{H}$ around $A^{0}+W e A^{1}$. This emphasizes the importance of pushing the Taylor expansion of $\mathcal{F}$ and $\mathcal{H}$ up to the order 1. 
LEMMA 3.4. We have

$$
\begin{aligned}
- & \frac{\omega(b+d)}{2 b} \frac{1}{W e}\left[\int_{\Omega} D \mathcal{F}\left(A^{0}+W e A^{1}\right)\left(A-A^{0}-W e A^{1}\right)\right. \\
& \left.+\frac{1}{W e} \int_{0}^{t} \int_{\Omega} D \mathcal{H}\left(A^{0}+W e A^{1}\right)\left(A-A^{0}-W e A^{1}\right)\right] \\
=- & \left.\frac{\omega(b+d)}{2 b} \frac{1}{W e} \int_{\Omega} D \mathcal{F}\left(A^{0}+W e A^{1}\right)\left(A-A^{0}-W e A^{1}\right)\right|_{t=0} \\
& -\int_{0}^{t} \int_{\Omega}\left[\tau: \nabla u^{0}+\tau^{0}: \nabla u\right]+2 \int_{0}^{t} \int_{\Omega} \tau^{0}: \nabla u^{0} \\
& -\frac{\omega(b+d)}{2 b} \int_{0}^{t} \int_{\Omega} \operatorname{Tr}\left(\frac{\left(\partial_{t}+u \cdot \nabla\right) A^{1}}{\left(A^{0}\right)^{2}}\left(A-A^{0}-W e A^{1}\right)\right) \\
& +\frac{2(b+d)}{b^{2}} \int_{0}^{t} \int_{\Omega} \operatorname{Tr}\left(A-A^{0}-W e A^{1}\right) \tau: \nabla u^{0} \\
& +\frac{3 \omega(b+d)}{2 b} \int_{0}^{t} \int_{\Omega} \operatorname{Tr}\left(\left(A-A^{0}-W e A^{1}\right) \frac{\left(A^{1}\right)^{2}}{\left(A^{0}\right)^{4}}\right) \\
& +O(W e) .
\end{aligned}
$$

Again, the proof relies on a computation of the total time derivative of the left hand side of (3.30) and on expansions in $W e$. The computations, however, are much more heavy than in the preceding lemma. We shall see that the terms of order $O\left(W e^{0}\right)$ in

$-\left(\partial_{t}+u \cdot \nabla\right) D \mathcal{F}\left(A^{0}+W e A^{1}\right)\left(A-A^{0}-W e A^{1}\right)-\frac{1}{W e} \int_{\Omega} D \mathcal{H}\left(A^{0}+W e A^{1}\right)\left(A-A^{0}-W e A^{1}\right)$

compensate each other, which is not a surprise as $D \mathcal{F}\left(A^{0}\right)=D \mathcal{H}\left(A^{0}\right)=0$. The crucial terms appear at order $O(W e)$. The fundamental trick we use in order to highlight the crossed terms $\int_{0}^{t} \int_{\Omega} \tau: \nabla u^{0}$ and $\int_{0}^{t} \int_{\Omega} \tau^{0}: \nabla u$ is to express $A$ in terms of $\tau$ and $\operatorname{Tr} A$ :

$$
A=\left(1-\frac{\operatorname{Tr} A}{b}\right)\left[W e \frac{b}{(b+d) \omega} \tau+I\right] .
$$

This is somewhat reminiscent of the change of variable $A=\frac{W e}{\omega} \tau+I$ for the corotational and Oldroyd-B systems (see [17]). Thanks to (3.31), we get

$$
\begin{aligned}
\left(\partial_{t}+u \cdot \nabla\right) A & =\nabla u A+A(\nabla u)^{T}-\frac{b}{(b+d) \omega} \tau \\
& =2\left(1-\frac{\operatorname{Tr} A}{b}\right) D(u)-\frac{b}{(b+d) \omega} \tau+W e \frac{b}{(b+d) \omega}\left(1-\frac{\operatorname{Tr} A}{b}\right)\left[\nabla u \tau+\tau(\nabla u)^{T}\right] .
\end{aligned}
$$

Moreover, we split the term

$$
2\left(1-\frac{\operatorname{Tr} A}{b}\right) D(u)=2\left(1-\frac{\operatorname{Tr} A^{0}}{b}\right) D(u)-2 \frac{\operatorname{Tr}\left(A-A^{0}-W e A^{1}\right)}{b} D(u),
$$

so that

$$
\begin{aligned}
\left(\partial_{t}+u \cdot \nabla\right) A & =\frac{b}{(b+d) \omega}(2 \omega D(u)-\tau) \\
& -2 \frac{\operatorname{Tr}\left(A-A^{0}-W e A^{1}\right)}{b} D(u)+W e \frac{b}{(b+d) \omega}\left(1-\frac{\operatorname{Tr} A}{b}\right)\left[\nabla u \tau+\tau(\nabla u)^{T}\right] .
\end{aligned}
$$


Proof. [Proof of Lemma 3.4] The proof consists in the calculation of

$$
\begin{array}{r}
-\left(\partial_{t}+u \cdot \nabla\right) D \mathcal{F}\left(A^{0}+W e A^{1}\right)\left(A-A^{0}-W e A^{1}\right) \\
-\frac{1}{W e} \int_{\Omega} D \mathcal{H}\left(A^{0}+W e A^{1}\right)\left(A-A^{0}-W e A^{1}\right)
\end{array}
$$

We expand this quantity in terms of $W e$. As expounded above, we are especially interested in the terms of order 1 . For these terms, we use the change of variable $(3.31)$ in a decisive way. All the terms of order $W e^{2}$ or more are put in the remainder. In the sequel, we first compute (3.34), then (3.35).

- We begin with the computation of $(3.34)$ :

$$
\begin{aligned}
& -\left(\partial_{t}+u \cdot \nabla\right) D \mathcal{F}\left(A^{0}+W e A^{1}\right)\left(A-A^{0}-W e A^{1}\right) \\
& =-\operatorname{We} \operatorname{Tr}\left(\frac{\left(\partial_{t}+u \cdot \nabla\right) A^{1}}{\left(A^{0}+W e A^{1}\right)^{2}}\left(A-A^{0}-W e A^{1}\right)\right) \\
& +\operatorname{Tr}\left(\left(A^{0}+W e A^{1}\right)^{-1}\left(\partial_{t}+u \cdot \nabla\right)\left(A-A^{0}-W e A^{1}\right)\right) \\
& -\frac{\operatorname{Tr}\left(\left(\partial_{t}+u \cdot \nabla\right)\left(A-A^{0}-W e A^{1}\right)\right)}{1-\frac{\operatorname{Tr} A^{0}}{b}}
\end{aligned}
$$

We now look closely at the two first terms appearing in the right hand side above.

$\triangleright$ Term $3.36 \mathrm{a})$ :

$$
\begin{aligned}
& -W e \operatorname{Tr}\left(\frac{\left(\partial_{t}+u \cdot \nabla\right) A^{1}}{\left(A^{0}+W e A^{1}\right)^{2}}\left(A-A^{0}-W e A^{1}\right)\right) \\
& =-W e \operatorname{Tr}\left(\frac{\left(\partial_{t}+u \cdot \nabla\right) A^{1}}{\left(A^{0}\right)^{2}}\left(A-A^{0}-W e A^{1}\right)\right) \\
& \quad-W e^{2} \sum_{k=1}^{\infty}(-1)^{k}(k+1) W e^{k-1} \operatorname{Tr}\left(\frac{\left(\partial_{t}+u \cdot \nabla\right) A^{1}}{\left(A^{0}\right)^{2}}\left(\frac{A^{1}}{A^{0}}\right)^{k}\left(A-A^{0}-W e A^{1}\right)\right) .
\end{aligned}
$$

$\triangleright \operatorname{Term}(3.36 \mathrm{~b})$

$$
\begin{aligned}
& \operatorname{Tr}\left(\left(A^{0}+W e A^{1}\right)^{-1}\left(\partial_{t}+u \cdot \nabla\right)\left(A-A^{0}-W e A^{1}\right)\right) \\
& =\operatorname{Tr}\left(\frac{\left(\partial_{t}+u \cdot \nabla\right)\left(A-A^{0}-W e A^{1}\right)}{A^{0}}\right) \\
& \quad-W e \operatorname{Tr}\left(\frac{\left(\partial_{t}+u \cdot \nabla\right) A}{\left(A^{0}\right)^{2}} A^{1}\right) \\
& \quad+W e^{2}\left[\sum_{k=2}^{\infty}(-1)^{k} W e^{k-2} \operatorname{Tr}\left(\left(\partial_{t}+u \cdot \nabla\right)\left(A-A^{0}-W e A^{1}\right) \frac{\left(A^{1}\right)^{k}}{\left(A^{0}\right)^{k+1}}\right)\right. \\
& \left.\quad+\operatorname{Tr}\left(\frac{\left(\partial_{t}+u \cdot \nabla\right) A^{1}}{\left(A^{0}\right)^{2}} A^{1}\right)\right] .
\end{aligned}
$$

Adding the terms of order $W e^{0}$ in $(3.36)$, we already see that

$$
\operatorname{Tr}\left(\frac{\left(\partial_{t}+u \cdot \nabla\right)\left(A-A^{0}-W e A^{1}\right)}{A^{0}}\right)-\frac{\operatorname{Tr}\left(\left(\partial_{t}+u \cdot \nabla\right)\left(A-A^{0}-W e A^{1}\right)\right)}{1-\frac{\operatorname{Tr} A^{0}}{b}}=0,
$$

which means that the zeroth-order term vanishes.

At the order $O(W e)$ in $(3.36)$, we have the terms

$$
-\operatorname{Tr}\left(\frac{\left(\partial_{t}+u \cdot \nabla\right) A^{1}}{\left(A^{0}\right)^{2}}\left(A-A^{0}-W e A^{1}\right)\right)-\operatorname{Tr}\left(\frac{\left(\partial_{t}+u \cdot \nabla\right) A}{\left(A^{0}\right)^{2}} A^{1}\right) .
$$


We concentrate on the second term in 3.39 . We rely on the identity (3.33):

$$
\begin{aligned}
-\operatorname{Tr}\left(\frac{\left(\partial_{t}+u \cdot \nabla\right) A}{\left(A^{0}\right)^{2}} A^{1}\right)=- & \frac{b}{(b+d) \omega} \operatorname{Tr}\left(\frac{2 \omega D(u)-\tau}{\left(A^{0}\right)^{2}} A^{1}\right) \\
& +2 \frac{\operatorname{Tr}\left(A-A^{0}-W e A^{1}\right)}{b} \operatorname{Tr}\left(\frac{D(u)}{\left(A^{0}\right)^{2}} A^{1}\right) \\
& -W e \frac{b}{(b+d) \omega}\left(1-\frac{\operatorname{Tr} A}{b}\right) \operatorname{Tr}\left(\frac{\nabla u \tau+\tau(\nabla u)^{T}}{\left(A^{0}\right)^{2}} A^{1}\right)
\end{aligned}
$$

Notice that

$$
\begin{aligned}
-\frac{b}{(b+d) \omega} \operatorname{Tr}\left(\frac{2 \omega D(u)-\tau}{\left(A^{0}\right)^{2}} A^{1}\right) & =\frac{2 b}{(b+d) \omega}(\tau-2 \omega D(u)): D\left(u^{0}\right) \\
& =\frac{2 b}{(b+d) \omega}\left[\tau: \nabla u^{0}-\tau^{0}: \nabla u\right]
\end{aligned}
$$

which contains some of the crossed terms appearing in $(3.30)$, though not with the right sign for $\tau: \nabla u^{0}$. The analysis of $(3.35$ makes it possible to recover all the crossed terms of $(3.30)$.

- Let us now expand 3.35 in powers of $W e$ :

$$
\begin{aligned}
& -\frac{1}{W e} D \mathcal{H}\left(A^{0}+W e A^{1}\right)\left(A-A^{0}-W e A^{1}\right) \\
& =-\frac{1}{W e} \frac{\operatorname{Tr}\left(A-A^{0}-W e A^{1}\right)}{\left(1-\frac{\operatorname{Tr} A^{0}}{b}\right)^{2}}+\frac{1}{W e} \operatorname{Tr}\left(\frac{A-A^{0}-W e A^{1}}{\left(A^{0}+W e A^{1}\right)^{2}}\right) \\
& =-2 \operatorname{Tr}\left(\left(A-A^{0}-W e A^{1}\right) \frac{A^{1}}{\left(A^{0}\right)^{3}}\right) \\
& \quad+3 W e \operatorname{Tr}\left(\left(A-A^{0}-W e A^{1}\right) \frac{\left(A^{1}\right)^{2}}{\left(A^{0}\right)^{4}}\right) \\
& \quad+W e^{2} \sum_{k=3}^{\infty}(-1)^{k}(k+1) W e^{k-3} \operatorname{Tr}\left(\left(A-A^{0}-W e A^{1}\right) \frac{\left(A^{1}\right)^{k}}{\left(A^{0}\right)^{k+2}}\right) .
\end{aligned}
$$

Yet, thanks to 3.31

$$
-2 \operatorname{Tr}\left(\left(A-A^{0}-W e A^{1}\right) \frac{A^{1}}{\left(A^{0}\right)^{3}}\right)=-2 \operatorname{Tr}\left(\frac{A A^{1}}{\left(A^{0}\right)^{3}}\right)+2 W e \operatorname{Tr}\left(\frac{\left(A^{1}\right)^{2}}{\left(A^{0}\right)^{3}}\right) .
$$

On the one hand,

$$
\begin{aligned}
& -2 \operatorname{Tr}\left(\frac{A A^{1}}{\left(A^{0}\right)^{3}}\right) \\
& =-4 W e\left(1-\frac{\operatorname{Tr} A}{b}\right) \frac{1}{\omega} \tau: D\left(u^{0}\right) \\
& =-4 W e \frac{b}{(b+d) \omega} \tau: \nabla u^{0}+4 W e \frac{\operatorname{Tr}\left(A-A^{0}-W e A^{1}\right)}{\omega b} \tau: \nabla u^{0},
\end{aligned}
$$

and on the other hand

$$
2 W e T r\left(\frac{\left(A^{1}\right)^{2}}{\left(A^{0}\right)^{3}}\right)=\frac{4 b}{\omega(b+d)} W e \tau^{0}: \nabla u^{0}
$$


We conclude that 3.30 holds. Notice that one uses ideas similar to those of the remark 6 in order to see that the remainder terms (3.37), (3.38) and $(3.40)$ are indeed of order $O(W e)$. To ascertain the convergence of the infinite series, one argues as in the remark 7 .

Gathering the estimates of the lemmas 3.2 , 3.3 and 3.4 we end up at the following estimate of the relative entropy: there exists $C>0$ such that for all $0<W e<1$,

$$
\begin{aligned}
C \mathfrak{E}(t) \leq \mathfrak{E}(0) & -\int_{0}^{t} \int_{\Omega}\left(U^{(r)} \cdot \nabla u^{0}\right) \cdot U^{(r)} \\
& -\frac{\omega(b+d)}{2 b} \int_{0}^{t} \int_{\Omega} \operatorname{Tr}\left(\frac{\left(\partial_{t}+u \cdot \nabla\right) A^{1}}{\left(A^{0}\right)^{2}}\left(A-A^{0}-W e A^{1}\right)\right) \\
& +\frac{2(b+d)}{b^{2}} \int_{0}^{t} \int_{\Omega} \operatorname{Tr}\left(A-A^{0}-W e A^{1}\right) \tau: \nabla u^{0} \\
& +\frac{3 \omega(b+d)}{2 b} \int_{0}^{t} \int_{\Omega} \operatorname{Tr}\left(\left(A-A^{0}-W e A^{1}\right) \frac{\left(A^{1}\right)^{2}}{\left(A^{0}\right)^{4}}\right) \\
& +O(W e) .
\end{aligned}
$$

We proceed for the term

$$
-\int_{0}^{t} \int_{\Omega}\left(U^{(r)} \cdot \nabla u^{0}\right) \cdot U^{(r)}
$$

exactly as we did for $\mathrm{A}$ in 2.15 , while proving the strong convergence of the corotational system. In dimension $d=3$, for instance, we bound for all $\nu>0$

$$
\left|\int_{0}^{t} \int_{\Omega}\left(U^{(r)} \cdot \nabla u^{0}\right) \cdot U^{(r)}\right| \leq \frac{1}{4 \nu^{3}} \int_{0}^{t}\left\|\nabla u^{0}\right\|_{L^{2}(\Omega)}^{4}\left\|U^{(r)}\right\|_{L^{2}(\Omega)}^{2}+\frac{3 \nu}{4} \int_{0}^{t}\left\|\nabla U^{(r)}\right\|_{L^{2}(\Omega)}^{2},
$$

so that for $\nu$ sufficiently small independent of $W e$, we can absorb the second term in the left hand side of (3.41).

REMARK 8 (On the dimension). Notice that this is the only step of our proof which is dependent on the dimension. In particular the computations of lemmas 3.2, 3.3 and 3.4 can be carried out for every $d \geq 2$.

For (3.41a), we first split the total time derivative according to 3.29 . We then estimate

$$
\begin{aligned}
& \left|\frac{\omega(b+d)}{2 b} \int_{0}^{t} \int_{\Omega} \operatorname{Tr}\left(\frac{\left(\partial_{t}+u^{0} \cdot \nabla\right) A^{1}}{\left(A^{0}\right)^{2}}\left(A-A^{0}-W e A^{1}\right)\right)\right| \\
& \leq \frac{\omega(b+d)}{4 b} \frac{W e^{2}}{\nu} \int_{0}^{t} \int_{\Omega}\left|\frac{\left(\partial_{t}+u^{0} \cdot \nabla\right) A^{1}}{\left(A^{0}\right)^{2}}\right|^{2}+\frac{\omega(b+d)}{4 b} \frac{\nu}{W e^{2}} \int_{0}^{t}\left\|A-A^{0}-W e A^{1}\right\|_{L^{2}(\Omega)}^{2}
\end{aligned}
$$

and absorb the second term in $(3.42)$ in the left hand side for $\nu$ small enough independently of $W e$ (see the Gronwall inequality (3.18)). Moreover,

$$
\begin{aligned}
& \left|\frac{\omega(b+d)}{2 b} \int_{0}^{t} \int_{\Omega} \operatorname{Tr}\left(\frac{\left(u-u^{0}-W e u^{1}\right) \cdot \nabla A^{1}}{\left(A^{0}\right)^{2}}\left(A-A^{0}-W e A^{1}\right)\right)\right| \\
& \leq \frac{\omega(b+d)}{4 b} \frac{W e^{2}}{\nu} \int_{0}^{t} \int_{\Omega}\left|\frac{\left(u-u^{0}-W e u^{1}\right) \cdot \nabla A^{1}}{\left(A^{0}\right)^{2}}\right|^{2}+\frac{\omega(b+d)}{4 b} \frac{\nu}{W e^{2}} \int_{0}^{t}\left\|A-A^{0}-W e A^{1}\right\|_{L^{2}(\Omega)}^{2},
\end{aligned}
$$

so that we absorb the second term in 3.43 in the left hand side of the Gronwall inequality, and absorb the first one in the integral term $\int_{0}^{t} \mathcal{C}(s) \mathfrak{D}(s) d s$. The term

$$
W e \frac{\omega(b+d)}{2 b} \int_{0}^{t} \int_{\Omega} \operatorname{Tr}\left(\frac{u^{1} \cdot \nabla A^{1}}{\left(A^{0}\right)^{2}}\left(A-A^{0}-W e A^{1}\right)\right)=O(W e)
$$


is put in the remainder. We handle the terms $3.41 \mathrm{~b})$ and $(3.41 \mathrm{c})$ similarly.

Finally, we conclude that the Gronwall inequality $(3.18)$ on $\mathfrak{D}$ is true:

$$
\mathfrak{D} \leq \mathfrak{E}(0)+\int_{0}^{t} \mathcal{C}(s) \mathfrak{D}(s) d s+O(W e)
$$

We do not make the function $s \mapsto \mathcal{C}(s)$ explicit. Note that it may depend on $b, \omega, u^{0}, u^{1}$, but not on $W e$. Moreover, as stated above, it is positive, and locally bounded. Let $T^{*}$ be the supremum, which may be infinite, of the times $T$ such that

$$
\sup _{0 \leq t<T} \int_{0}^{t} \mathcal{C}(s) d s<\infty .
$$

We infer from (3.44) that for all $T>0$, for all $0 \leq t<T$,

$$
0 \leq \mathfrak{D}(t) \leq[\mathfrak{E}(0)+O(W e)] \exp \left(t+\int_{0}^{t} \mathcal{C}(s) d s\right) .
$$

This, along with (3.22a) and (3.22b), implies the estimates 1.15 and finishes the proof of Theorem 1.4 .

\subsection{Final remarks.}

On the Giesekus and PTT models. In the paper [26, N. Masmoudi addresses the existence of weak solutions for two other systems, namely the Giesekus model and the Phan-Thien and Tannes (PTT) model. These models are quite similar, so we just give some indications for Giesekus's. The non-dimensional equation on the stress tensor reads

$$
\partial_{t} \tau+u \cdot \nabla \tau-\nabla u \tau-\tau(\nabla u)^{T}+\alpha \tau^{2}+\frac{1}{W e} \tau=\frac{2 \omega}{W e} D(u)
$$

with $\alpha>0$. As usually, we can rewrite the equation 3.45 in terms of the positive definite matrix $A:=I+\frac{W e}{\omega} \tau:$

$$
\partial_{t} A+u \cdot \nabla A-\nabla u A-A(\nabla u)^{T}+\frac{\alpha \omega^{2}}{W e^{2}}(A-I)^{2}+\frac{\omega}{W e^{2}}(A-I)=0 .
$$

Computing,

$$
\begin{aligned}
\left(\partial_{t}+u \cdot \nabla\right)(\operatorname{det} A)^{\frac{1}{d}} & =-\frac{\alpha \omega^{2}}{W e^{2}} \frac{1}{d}(\operatorname{det} A)^{\frac{1}{d}} \operatorname{Tr}\left(A^{-1}(A-I)^{2}\right)-\frac{\omega}{W e^{2}} \frac{1}{d}(\operatorname{det} A)^{\frac{1}{d}} \operatorname{Tr}\left(A^{-1}(A-I)\right) \\
& \geq \frac{\omega}{W e^{2}}(\operatorname{det} A)^{\frac{1}{d}}\left(\frac{\operatorname{Tr}\left(A^{-1}\right)}{d}-1\right) \\
& \geq \frac{\omega}{W e^{2}}\left(1-(\operatorname{det} A)^{\frac{1}{d}}\right),
\end{aligned}
$$

we infer that the property $\operatorname{det} A \geq 1$ is propagated in time, so that $\operatorname{Tr} A \geq d$ i.e. $\operatorname{Tr} \tau \geq 0$. Assume now that $\operatorname{det} A_{0} \geq 1$. Then, we have the following free energy decay in time

$$
\begin{aligned}
& \frac{1}{2}\|u(t, \cdot)\|_{L^{2}(\Omega)}+(1-\omega) \int_{0}^{t} \int_{\Omega}\|\nabla u\|_{L^{2}(\Omega)} \\
& \quad+\frac{\omega}{2 W e} \int_{\Omega} \operatorname{Tr} \tau(t, \cdot)+\frac{\omega}{2 W e} \int_{0}^{t} \int_{\Omega}\left(\alpha|\tau|^{2}+\frac{1}{W e} \operatorname{Tr} \tau\right) \\
& \leq \frac{1}{2}\left\|u_{0}\right\|_{L^{2}(\Omega)}+\frac{\omega}{2 W e} \int_{\Omega} \operatorname{Tr} \tau_{0} .
\end{aligned}
$$

One immmediately deduces a $L^{2}((0, T) \times \Omega)$ bound on $\tau$. 
We formally see that $\tau$ (resp. $A$ ) tends to $2 \omega D\left(u^{0}\right)$ (resp. $I$ ), when $W e \rightarrow 0$. To address the asymptotics, one works with the entropy expressed in terms of $A$

$$
\begin{aligned}
& \frac{1}{2}\|u(t, \cdot)\|_{L^{2}(\Omega)}+(1-\omega) \int_{0}^{t} \int_{\Omega}\|\nabla u\|_{L^{2}(\Omega)} \\
& \quad+\frac{\omega}{2 W e} \int_{\Omega}[\operatorname{Tr} A(t, \cdot)-d]+\frac{\omega^{2}}{2 W e^{3}} \int_{0}^{t} \int_{\Omega}\left[\alpha \omega|A-I|^{2}+\operatorname{Tr} A-d\right] \\
& \leq \frac{1}{2}\left\|u_{0}\right\|_{L^{2}(\Omega)}+\frac{\omega}{2 W e} \int_{\Omega}\left(\operatorname{Tr} A_{0}-d\right) .
\end{aligned}
$$

As in the FENE-P case, one builds corrector terms of $u, A$ and $\tau$. Evidently, $u^{0}$ is the solution of the Navier-Stokes system with no-slip boundary condition and initial data equal to $u_{0}, A^{0}:=I$ and $\tau^{0}:=2 \omega D\left(u^{0}\right)$. One derives a formula for the relative entropy by expanding the free energy (3.48) around $A^{0}+W e A^{1}$. The fundamental remark is that $A \mapsto|A-I|^{2}$ is globally convex. The computations are far more simple than for the FENE-P system and lead to the convergence in the relative entropy norm

$$
\begin{array}{r}
\sup _{t \in[0, T]}\left(\frac{1}{2}\left\|u-u^{0}-W e u^{1}\right\|_{L^{2}(\Omega)}^{2}+(1-\omega) \int_{0}^{t} \int_{\Omega}\left\|\nabla\left(u-u^{0}-W e u^{1}\right)\right\|_{L^{2}(\Omega)}^{2}\right. \\
\left.\quad+\frac{\alpha \omega^{3}}{2 W e^{3}} \int_{0}^{t}\left\|A-A^{0}-W e A^{1}\right\|_{L^{2}(\Omega)}^{2}\right)^{\frac{1}{2}} .
\end{array}
$$

Further details are left to the reader.

On the Oldroyd-B system. For the Oldroyd-B system, the constitutive equation is (3.45) with $\alpha=0$. As for the Giesekus model, the property det $A_{0} \geq 1$ propagates in time and implies $\operatorname{Tr} \tau=0$. Instead of (3.47), we have

$$
\begin{aligned}
\frac{1}{2}\|u(t, \cdot)\|_{L^{2}(\Omega)}+(1-\omega) \int_{0}^{t} \int_{\Omega}\|\nabla u\|_{L^{2}(\Omega)}+\frac{\omega}{W e} \int_{\Omega} \operatorname{Tr} \tau(t, \cdot)+ & \frac{\omega}{W e^{2}} \int_{0}^{t} \int_{\Omega} \operatorname{Tr} \tau \\
& \leq \frac{1}{2}\left\|u_{0}\right\|_{L^{2}(\Omega)}+\frac{\omega}{W e} \int_{\Omega} \operatorname{Tr} \tau_{0}
\end{aligned}
$$

so that we lack an a priori bound on $\tau$ in $L^{2}((0, T) \times \Omega)$. The Cauchy theory for weak solutions of this system is lacunary. So is the analysis of the Newtonian limit (for an Oldroyd-B system with a regularizing term, see the analysis of J. Barrett and S. Boyaval [1]). We are not able to say anything on the limit, even on a formal level assuming the existence of solutions. As the Oldroyd-B system is widely used in applications, despite its inadequate prediction of some physical phenomena, these questions are challenging.

\section{Appendix A. A remark on the Newtonian limit for oscillating initial data.}

This appendix is devoted to the proof of Proposition $1.1 \mathrm{bis}$. We assume that the initial data $\tau_{0, n}$ is strongly oscillating in $n$ (not in $W e$ ). In our setting (see below), these oscillations introduce defect measures at the limit $n \rightarrow \infty$. In order to handle these defect measures, we need refined a priori bounds on the solutions of (1.1). Our analysis is close to the paper [24] by P.-L. Lions and N. Masmoudi, where the existence of weak solutions is proved.

A.1. Further a priori bounds for the corotational system. We complement the bound (1.3) by carrying out $L^{p}\left((0, T), L^{q}\right)$ estimates on the system (1.1). In particular, these bounds are crucial for the Cauchy theory of weak solutions as developed in [24].

The proof of such estimates is divided into two parts. First, assuming an $L^{p}\left((0, T), L^{q}\right)$ estimate on $\tau$, we prove a control on the velocity using the momentum equation. Then, using the equation on $\tau$, we use a Gronwall lemma to show an $L^{p}\left((0, T), L^{q}\right)$ estimate on $\tau$ taking advantage of the estimate of the velocity in terms of $\tau$. 
A.1.1. An $L^{p}\left((0, T) ; L^{q}\right)$ control of the velocity field $u$ through an $L^{p}\left((0, T) ; L^{q}\right)$ estimate on $\tau$. In order to estimate $\nabla u$ in $L^{p}\left((0, T) ; L^{q}\right)$, we decompose $u$ in a sum $u=u^{1}+u^{2}+u^{3}$, where $u^{1}$ and $u^{2}$ are the unique solutions to the Stokes system

$$
\left\{\begin{array}{rl}
\partial_{t} u^{i}-\nu \Delta u^{i}+\nabla p^{i} & =f^{i} \\
\nabla \cdot u^{i} & =0 \\
u^{i}(0, \cdot) & =0
\end{array},\right.
$$

with $f^{1}:=-u \cdot \nabla u, f^{2}:=\nabla \cdot \tau$, and where $u^{3}$ is the unique solution to

$$
\left\{\begin{array}{rl}
\partial_{t} u^{3}-\nu \Delta u^{3}+\nabla p^{3} & =0 \\
\nabla \cdot u^{3} & =0 \\
u^{3}(0, \cdot) & =u_{0}
\end{array} .\right.
$$

As $u_{0}$ belongs to $I_{p, q}$ for $4 \leq q<\infty$ and $1<p \leq \frac{q}{q-1}$,

$$
\left\|\nabla u^{3}\right\|_{L^{p}\left((0, T) ; L^{q}\right)} \leq C\left\|u_{0}\right\|_{I_{p, q}} .
$$

The second term $u^{2}$ can be estimated by applying the nonstationary version of Cattabriga's estimate given in [13] (see corollary 4.2 and estimate (1.6)):

$$
\left\|\nabla u^{2}\right\|_{L^{p}\left((0, T) ; L^{q}\right)} \leq C\|\tau\|_{L^{p}\left((0, T) ; L^{q}\right)} .
$$

From 1.3, we conclude that $u \cdot \nabla u$ is bounded in $L^{r}\left((0, T) ; L^{s}\right)$ for all $1 \leq r \leq 2$ and $s=\frac{2 r}{3 r-2}$. Hence, applying Theorem 2.8 from [14] and Sobolev's injection theorem, we get

$$
\left\|\nabla u^{1}\right\|_{L^{r}\left((0, T) ; L^{s^{\star}}\right)} \leq C\left\|\nabla^{2} u^{1}\right\|_{L^{r}\left((0, T) ; L^{s}\right)} \leq C\|u \cdot \nabla u\|_{L^{r}\left((0, T) ; L^{s}\right)} \leq C\left[r, s,\left\|u_{0}\right\|_{L^{2}},\left\|\tau_{0}\right\|_{L^{2}}\right]
$$

with $1<r \leq 2$ and $s^{\star}=\frac{2 s}{2-s}=\frac{r}{r-1}$, so that

$$
\left\|\nabla u^{1}\right\|_{L^{\frac{q}{q-1}}\left((0, T) ; L^{q}\right)} \leq C\left[\left\|u_{0}\right\|_{L^{2}},\left\|\tau_{0}\right\|_{L^{2}}\right] .
$$

From A.3, A.4 and A.5 we conclude that

$$
\|\nabla u\|_{L^{\frac{q}{q-1}\left((0, T) ; L^{q}\right)}} \leq C\|\tau\|_{L^{\frac{q}{q-1}}\left((0, T) ; L^{q}\right)}+C\left[\left\|u_{0}\right\|_{L^{2}},\left\|u_{0}\right\|_{I_{p, q}},\left\|\tau_{0}\right\|_{L^{2}}\right],
$$

with constants uniform in $T$ and $W e$.

A.1.2. Extra $L^{p}\left((0, T) ; L^{q}\right)$ estimates on $\tau$ through a Gronwall estimate. Let us now estimate $\|\tau\|_{L^{p}\left((0, T) ; L^{q}\right)}$, for any $0 \leq T$,

$$
1 \leq p \leq \frac{q}{q-1} \quad \text { and } \quad 3<q<\infty .
$$

Testing the equation on $\tau$ against $\tau|\tau|^{q-2}$ gives

$$
\begin{array}{r}
\frac{1}{q} \partial_{t}\|\tau\|_{L^{q}}^{q}+\int_{\mathbb{R}^{2}}(u \cdot \nabla \tau): \tau|\tau|^{q-2}+\int_{\mathbb{R}^{2}} \tau W(u) \cdot \tau|\tau|^{q-2}-\int_{\mathbb{R}^{2}} W(u) \tau: \tau|\tau|^{q-2}+\frac{1}{W e}\|\tau\|^{q} \\
=\frac{2 \omega}{W e} \int_{\mathbb{R}^{2}} D(u): \tau|\tau|^{q-2}
\end{array}
$$

Yet, it follows from the incompressibility of the velocity field

$$
\begin{aligned}
\int_{\mathbb{R}^{2}}(u \cdot \nabla \tau): \tau|\tau|^{q-2} & =\int_{\mathbb{R}^{2}} u_{\alpha}\left(\partial_{\alpha} \tau_{\beta \gamma}\right) \tau_{\beta \gamma}|\tau|^{q-2} \\
& =\int_{\mathbb{R}^{2}} u_{\alpha} \frac{1}{q} \partial_{\alpha}\left(|\tau|^{q}\right)=\int_{\mathbb{R}^{2}} \partial_{\alpha}\left(u_{\alpha} \frac{|\tau|^{q}}{q}\right)=0,
\end{aligned}
$$


and

$$
\begin{aligned}
\int_{\mathbb{R}^{2}} \tau W(u): \tau|\tau|^{q-2} & =\int_{\mathbb{R}^{2}} \tau_{\alpha \gamma} W(u)_{\gamma \beta} \tau_{\alpha \beta}|\tau|^{q-2} \\
& =\frac{1}{2}\left[\int_{\mathbb{R}^{2}} \tau_{\alpha \gamma} \partial_{\gamma} u_{\beta} \tau_{\alpha \beta}|\tau|^{q-2}-\int_{\mathbb{R}^{2}} \tau_{\alpha \gamma} \partial_{\gamma} u_{\beta} \tau_{\alpha \beta}|\tau|^{q-2}\right]=0 .
\end{aligned}
$$

Therefore,

$$
\frac{1}{q} \partial_{t}\|\tau\|_{L^{q}}^{q}+\frac{1}{W e}\|\tau\|_{L^{q}}^{q}=\frac{2 \omega}{W e} \int_{\mathbb{R}^{2}} D(u): \tau|\tau|^{q-2} \leq \frac{2 \omega}{W e}\|\nabla u\|_{L^{q}}\|\tau\|_{L^{q}}^{q-1} .
$$

A.1.3. Gronwall Lemma related to $\tau$. Letting $Z:=\|\tau\|_{L^{q}}^{q}$, it follows from A.8,

$$
\frac{1}{q} \frac{\partial_{t} Z}{Z^{\frac{q-1}{q}}}+\frac{1}{W e} Z^{\frac{1}{q}} \leq \frac{2 \omega}{W e}\|\nabla u\|_{L^{q}}
$$

which rewrites

$$
\partial_{t}\left(Z^{\frac{1}{q}}\right)+\frac{1}{W e} Z^{\frac{1}{q}} \leq \frac{2 \omega}{W e}\|\nabla u\|_{L^{q}} .
$$

A Gronwall-type argument yields for all $t>0$,

$$
\|\tau\|_{L^{q}}(t)=Z^{\frac{1}{q}}(t) \leq\|\tau\|_{L^{q}}(0) \exp \left(-\frac{t}{W e}\right)+\frac{2 \omega}{W e} \int_{0}^{t}\|\nabla u\|_{L^{q}}(s) \exp \left(\frac{s-t}{W e}\right) d s .
$$

On the one hand, for all $0<t \leq T$,

$$
\begin{aligned}
& \left\|\int_{0}^{t}\right\| \nabla u\left\|_{L^{q}}(s) \exp \left(\frac{s-t}{W e}\right) d s\right\|_{L^{\infty}(0, T)} \leq C W e^{\frac{1}{q}}\|\nabla u\|_{L^{\frac{q}{q-1}}\left((0, T) ; L^{q}\right)} \\
& \leq C W e^{\frac{1}{q}}\|\tau\|_{L^{\frac{q}{q-1}}\left((0, T) ; L^{q}\right)}+W e^{\frac{1}{q}} C\left[\left\|u_{0}\right\|_{L^{2}},\left\|u_{0}\right\|_{I_{p, q}},\left\|\tau_{0}\right\|_{L^{2}}\right]
\end{aligned}
$$

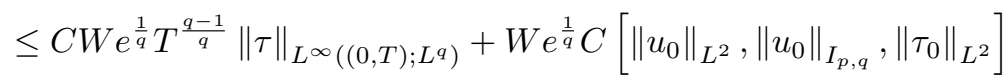

which implies for $T_{1}$ sufficiently small

$$
\|\tau\|_{L^{\infty}\left(\left(0, T_{1}\right) ; L^{q}\right)} \leq \frac{1}{1-2 \omega\left(\frac{T_{1}}{W e}\right)^{\frac{q}{q-1}}}\|\tau\|_{L^{q}}(0)+\frac{C\left[\left\|u_{0}\right\|_{L^{2}},\left\|u_{0}\right\|_{I_{p, q}},\left\|\tau_{0}\right\|_{L^{2}}\right]}{W e^{\frac{q-1}{q}}\left(1-2 \omega\left(\frac{T_{1}}{W e}\right)^{\frac{q}{q-1}}\right)} .
$$

The latter yields a local in time a priori estimate on $\tau$ and then on $\nabla u$ using the first part: for all $T>0$,

$$
\|\tau\|_{L^{\infty}\left((0, T) ; L^{q}\left(\mathbb{R}^{2}\right)\right)} \leq C\left[T, W e,\left\|u_{0}\right\|_{L^{2}},\left\|u_{0}\right\|_{I_{p, q}},\left\|\tau_{0}\right\|_{L^{2}},\left\|\tau_{0}\right\|_{L^{q}}\right]
$$

and

$$
\|\nabla u\|_{L^{\frac{q}{q-1}}\left((0, T) ; L^{q}\left(\mathbb{R}^{2}\right)\right)} \leq C\left[T, W e,\left\|u_{0}\right\|_{L^{2}},\left\|u_{0}\right\|_{I_{p, q}},\left\|\tau_{0}\right\|_{L^{2}},\left\|\tau_{0}\right\|_{L^{q}}\right] .
$$

The explicit computation of the constants appearing in A.11 and A.12 is tedious. Note that they blow up exponentially fast when $T \rightarrow \infty$ or $W e \rightarrow 0$.

On the other hand,

$$
\begin{aligned}
\left\|\int_{0}^{t}\right\| \nabla u\left\|_{L^{q}}(s) \exp \left(\frac{s-t}{W e}\right) d s\right\|_{L^{1}(0, T)} & \leq \int_{0}^{T}\|\nabla u\|_{L^{q}}(s) \int_{s}^{T} \exp \left(\frac{s-T}{W e}\right) d t d s \\
& \leq W e\|\nabla u\|_{L^{1}\left((0, T) ; L^{q}\right)}
\end{aligned}
$$


and

$$
\left\|\int_{0}^{t}\right\| \nabla u\left\|_{L^{q}}(s) \exp \left(\frac{s-t}{W e}\right) d s\right\|_{L^{\infty}(0, T)} \leq W e\|\nabla u\|_{L^{\infty}\left((0, T) ; L^{q}\right)},
$$

from which we get by interpolation

$$
\left\|\int_{0}^{t}\right\| \nabla u\left\|_{L^{q}}(s) \exp \left(\frac{s-t}{W e}\right) d s\right\|_{L^{\frac{q}{q-1}}(0, T)} \leq W e\|\nabla u\|_{L^{\frac{q}{q-1}}\left((0, T) ; L^{q}\right)} .
$$

Combining this last inequality with A.9 yields

$$
\|\tau\|_{L^{\frac{q}{q-1}}\left((0, T) ; L^{q}\right)} \leq W e^{\frac{q-1}{q}}\|\tau\|_{L^{q}}(0)+2 \omega\|\nabla u\|_{L^{\frac{q}{q-1}}\left((0, T) ; L^{q}\right)},
$$

and for $\omega$ sufficiently small, thanks to (A.6), a bound

$$
\|\tau\|_{L^{\frac{q}{q-1}}\left((0, \infty) ; L^{q}\right)} \leq C\left[\left\|u_{0}\right\|_{L^{2}},\left\|u_{0}\right\|_{I_{p, q}},\left\|\tau_{0}\right\|_{L^{2}},\left\|\tau_{0}\right\|_{L^{q}}\right]
$$

uniform in time and in $W e$.

REMARK 9. The singularity in $1 / W e^{\frac{q-1}{q}}$ in the estimate A.10 prevents us from obtaining a bound uniform in We in $L_{l o c}^{\infty}\left((0, \infty) ; L^{q}\right)$. However, for fixed $W e$, we can use this bound. This explains why this bound is usefull for the proof of weak solutions, and not for the low Weissenberg asymptotic analysis.

A.2. Newtonian limit with defect measures in the initial data. Let $\left(u_{n}, \tau_{n}\right)$ be a sequence of weak solutions to (1.1) associated to the initial conditions

$$
u_{n}(0, \cdot):=u_{0, n}(\cdot), \quad \tau_{n}(0, \cdot):=\tau_{0, n}(\cdot)
$$

We assume that:

- The sequence $\left(u_{n}, \tau_{n}\right)$ satisfies 1.3 and the a priori bounds (A.11) and A.12. Note that the energy bound $(1.3)$ bounds $u_{n}$ (resp. $\tau_{n}$ ) uniformly in $n$ and $W e$ in the spaces $L^{2}\left((0, \infty) ; \dot{H}^{1}\right), L^{\infty}\left((0, \infty) ; L^{2}\right)\left(\operatorname{resp} . L^{2}\left((0, \infty) ; L^{2}\right)\right)$.

- $u_{0, n}$ converges strongly in $L^{2}$ toward $u_{0}$.

- $\tau_{0, n}$ is uniformly equiintegrable in $L^{2}$, i.e. that

$$
\sup _{n} \int_{\left|\tau_{0, n}\right| \geq M}\left|\tau_{0, n}\right|^{2} \stackrel{M \rightarrow \infty}{\longrightarrow} 0 .
$$

In particular, we do not assume that $\tau_{0, n}$ converges strongly in $L^{2}$, which allows the presence of defect measures initially.

A.2.1. Defect measures. We begin our analysis by making a change of unknown, underlining some special features of $(1.1)$ when $d=2$. Following [6, equation (251)], let us introduce the new unknowns

$$
a_{n}:=\tau_{n, 11}-\tau_{n, 22}, \quad b_{n}:=\tau_{n, 12}, \quad c_{n}:=\tau_{n, 11}+\tau_{n, 22} .
$$

We compute (dropping for the moment the subscripts $n$ )

$$
\tau W(u)-W(u) \tau=\left(\begin{array}{cc}
\tau_{12}\left(\partial_{2} u_{1}-\partial_{1} u_{2}\right) & \frac{1}{2}\left(\tau_{11}-\tau_{22}\right)\left(\partial_{1} u_{2}-\partial_{2} u_{1}\right) \\
\frac{1}{2}\left(\tau_{11}-\tau_{22}\right)\left(\partial_{1} u_{2}-\partial_{2} u_{1}\right) & \tau_{12}\left(\partial_{1} u_{2}-\partial_{2} u_{1}\right)
\end{array}\right) .
$$

Hence, the transport equation on $\tau_{n}$ becomes

$$
\begin{cases}\partial_{t} a_{n}+u_{n} \cdot \nabla a_{n}-2 b_{n} \text { curlu }_{n}+\frac{a_{n}}{W e} & =\frac{2 \omega}{W e}\left(\partial_{1} u_{n, 1}-\partial_{2} u_{n, 2}\right) \\ \partial_{t} b_{n}+u_{n} \cdot \nabla b_{n}+\frac{1}{2} a_{n} \text { curlu }_{n}+\frac{b_{n}}{W e} & =\frac{\omega}{W e}\left(\partial_{1} u_{n, 2}+\partial_{2} u_{n, 1}\right) \\ \partial_{t} c_{n}+u_{n} \cdot \nabla c_{n}+\frac{c_{n}}{W e} & =0\end{cases}
$$


where $\operatorname{curl}_{n}:=\partial_{1} u_{n, 2}-\partial_{2} u_{n, 1}$. In particular $c_{n}$ is decoupled from $a_{n}$ and $b_{n}$. Of course, the sequences $\left(a_{n}\right),\left(b_{n}\right)$ and $\left(c_{n}\right)$ inherit from the properties of $\left(\tau_{n}\right)$, and $\left(a_{n}\right)$ (resp. $\left.\left(b_{n}\right),\left(c_{n}\right)\right)$ satisfy the a priori bounds (1.3) and A.11).

The presence of defect measures in the initial data means that

$$
\begin{gathered}
\left|a_{0, n}\right|^{2} \stackrel{*}{\rightarrow}\left|a_{0}\right|^{2}+\alpha_{0} L^{\infty}\left((0, T) ; L^{\frac{q}{2}}\right), \\
\left|b_{0, n}\right|^{2} \stackrel{*}{*}\left|b_{0}\right|^{2}+\beta_{0} L^{\infty}\left((0, T) ; L^{\frac{q}{2}}\right) .
\end{gathered}
$$

A way to quantify the possible loss of convergence in products of weakly converging sequences is to introduce defect measures. As

$$
\begin{array}{rll}
\qquad\left|a_{n}\right|^{2} \text { is uniformly bounded in } n \text { in (u.b.) } L^{\infty}\left((0, T) ; L^{\frac{q}{2}}\right), & L^{\infty}\left((0, T) ; L^{\frac{q}{2}}\right), \\
\left|b_{n}\right|^{2} & \text { u.b. in } & L^{\frac{q}{q-1}}\left((0, T) ; L^{\frac{q}{2}}\right), \\
a_{n} \text { curlu }_{n} & \text { u.b. in } & L^{\frac{q}{q-1}}\left((0, T) ; L^{\frac{q}{2}}\right), \\
b_{n} \text { curlu }_{n} & \text { u.b. in } & L^{\frac{q}{q-1}}\left((0, T) ; L^{\frac{q}{2}}\right), \\
\left(\partial_{1} u_{1, n}-\partial_{2} u_{2, n}\right) a_{n} & \text { u.b. in } & L^{2}\left((0, T) ; L^{\frac{q}{2}}\right), \\
\left(\partial_{1} u_{2, n}+\partial_{2} u_{1, n}\right) b_{n} & \text { u.b. in } & L^{1}\left((0, \infty) ; L^{1}\right), \\
\left|\nabla u_{n}\right|^{2} & \text { u.b. in }
\end{array}
$$

there exists $\alpha \in L_{l o c}^{\infty}\left((0, \infty) ; L^{\frac{q}{2}}\right)$ (resp. $\beta \in L_{l o c}^{\infty}\left((0, \infty) ; L^{\frac{q}{2}}\right), \delta, \varepsilon, \eta, \lambda \in L_{l o c}^{\frac{q}{q-1}}\left((0, \infty) ; L^{\frac{q}{2}}\right)$, $\left.\mu \in\left[L^{\infty}\left((0, \infty) ; L^{\infty}\right)\right]^{\prime}\right)$ such that for all $T>0$,

$$
\begin{array}{cc}
\left|a_{n}\right|^{2} \stackrel{*}{\rightarrow}|a|^{2}+\alpha & L^{\infty}\left((0, T) ; L^{\frac{q}{2}}\right), \\
\left|b_{n}\right|^{2} \stackrel{*}{\rightarrow}|b|^{2}+\beta & L^{\infty}\left((0, T) ; L^{\frac{q}{2}}\right), \\
a_{n} \text { curlu }_{n} \rightarrow \text { acurlu }+\delta & L^{\frac{q}{q-1}}\left((0, T) ; L^{\frac{q}{2}}\right), \\
b_{n} \text { curlu }_{n} \rightarrow \text { ccurlu }+\varepsilon & L^{\frac{q}{q-1}}\left((0, T) ; L^{\frac{q}{2}}\right), \\
\left(\partial_{1} u_{1, n}-\partial_{2} u_{2, n}\right) a_{n} \rightarrow\left(\partial_{1} u^{1}-\partial_{2} u^{2}\right) a+\eta L^{\frac{q}{q-1}}\left((0, T) ; L^{\frac{q}{2}}\right), \\
\left(\partial_{1} u_{2, n}+\partial_{2} u_{1, n}\right) b_{n} \rightarrow\left(\partial_{1} u^{2}+\partial_{2} u^{1}\right) b+\lambda L^{\frac{q}{q-1}}\left((0, T) ; L^{\frac{q}{2}}\right), \\
\left|\nabla u_{n}\right|^{2} \stackrel{*}{\rightarrow}|\nabla u|^{2}+\mu & {\left[L^{\infty}\left((0, \infty) ; L^{\infty}\right)\right]^{\prime} .}
\end{array}
$$

Let us state a couple of straightforward properties on the defect measures:

- The measures $\alpha, \beta, \mu$ are positive.

- For every bounded measurable set $E \subset(0, \infty) \times \mathbb{R}^{2}$,

$$
\begin{array}{ll}
|\eta(E)| \leq \sqrt{\mu(E)} \sqrt{\alpha(E)}, & |\lambda(E)| \leq \sqrt{\mu(E)} \sqrt{\beta(E)}, \\
|\delta(E)| \leq \sqrt{\mu(E)} \sqrt{\alpha(E)}, & |\varepsilon(E)| \leq \sqrt{\mu(E)} \sqrt{\beta(E)} .
\end{array}
$$

Moreover, testing the momentum equation against $u_{n}$ then passing to the limit, and passing to the limit in the momentum equation then testing against $u$ yields an equality between the terms appearing in the averaging process:

$$
2(1-\omega) \mu+\eta+2 \lambda=0 .
$$

Such an inequality implies in particular $\mu \in L_{l o c}^{2}\left((0, \infty) ; L^{\frac{2 q}{q+2}}\right)$.

A.2.2. Weak convergence analysis. Our purpose is to pass to the limit on $n$, then on $W e$. There are three steps:

1. We show the convergence of $u_{n}$ (resp. $\tau_{n}$ ) toward $u$ (resp. $\tau$ ), and pass to the weak limit $n \rightarrow \infty$ in the system 1.1 .

2. We pass to the limit $n \rightarrow \infty$ in 1.3 , in order to get a priori bounds on $u$ and $\tau$ uniform in $W e$.

3. We study the limit $W e \rightarrow 0$, as in the Section 2.1.

We will have recourse to the uniform equiintegrability of $\tau_{n}$ in $L_{l o c}^{\infty}\left((0, \infty) ; L^{2}\right)$ showed by P.-L. Lions and N. Masmoudi in 24]. 
First step. Classical arguments yield the existence of

$$
u \in L^{\infty}\left((0, \infty) ; L^{2, \sigma}\right) \cap L^{2}\left((0, \infty) ; \dot{H}^{1}\right) \quad \text { and } \quad \tau \in L^{\infty}\left((0, \infty) ; L^{2}\right) \cap L_{l o c}^{\infty}\left((0, \infty) ; L^{q}\right),
$$

satisfying the energy inequality $(1.3)$ with a right hand side slightly modified to account for the fact that $\tau_{0, n}$ does not converge to $\tau_{0}$ in $L^{2}$. Passing to the limit $n \rightarrow \infty$ in the system (1.1) for $\left(u_{n}, \tau_{n}\right)$, we get that $(u, \tau)$ solves

$$
\left\{\begin{aligned}
\partial_{t} u+u \cdot \nabla u-(1-\omega) \Delta u+\nabla p & =\nabla \cdot \tau \\
\nabla \cdot u & =0 \\
\partial_{t} \tau+u \cdot \nabla \tau+\tau W(u)-W(u) \tau+\left(\begin{array}{cc}
-\varepsilon & \frac{1}{2} \delta \\
-\frac{1}{2} \delta & \varepsilon
\end{array}\right)+\frac{1}{W e} \tau & =\frac{2 \omega}{W e} D(u)
\end{aligned}\right.
$$

in the sense of distributions. We focus now on the low Weissenberg limit in A.19.

Second step. We intend to let $n \rightarrow \infty$ in the energy estimate

$$
\begin{aligned}
\omega\left\|u_{n}\right\|_{L^{2}}^{2}+2 \omega(1-\omega) \int_{0}^{T}\left\|\nabla u_{n}\right\|_{L^{2}}^{2}+\frac{W e}{2}\left\|\tau_{n}\right\|_{L^{2}}^{2}+\int_{0}^{T}\left\|\tau_{n}\right\|_{L^{2}}^{2} & =\omega\left\|u_{0, n}\right\|_{L^{2}}^{2}+\frac{W e}{2}\left\|\tau_{0, n}\right\|_{L^{2}}^{2} \\
& =\omega
\end{aligned}
$$

so as to retrieve bounds on the defect measures $\delta$ and $\varepsilon$ uniform in $W e$. Assuming no defect measures initially (i.e. $\alpha_{0}=\beta_{0}=0$ ), we already know that $(1.3)$ is satisfied at the limit. Nevertheless, in the presence of defect measures, using the inequalities

$$
\begin{aligned}
\|u\|_{L^{\infty}\left((0, \infty) ; L^{2}\right)} & \leq \liminf _{n}\left\|u_{n}\right\|_{L^{\infty}\left((0, \infty) ; L^{2}\right)}, \\
\|\nabla u\|_{L^{2}\left((0, \infty) ; L^{2}\right)} & \leq \liminf _{n}\left\|\nabla u_{n}\right\|_{L^{2}\left((0, \infty) ; L^{2}\right)} \\
\|\tau\|_{L^{\infty}\left((0, \infty) ; L^{2}\right)} & \leq \liminf _{n}\left\|\tau_{n}\right\|_{L^{\infty}\left((0, \infty) ; L^{2}\right)}, \\
\|\tau\|_{L^{2}\left((0, \infty) ; L^{2}\right)} & \leq \liminf _{n}\left\|\tau_{n}\right\|_{L^{2}\left((0, \infty) ; L^{2}\right)},
\end{aligned}
$$

is responsible for the loss of a lot of information. In particular, we lose all information on the defect measures propagation.

The heart of the matter is to justify the following formal limit $n \rightarrow \infty$ in A.20

$$
\begin{aligned}
& \omega\|u\|_{L^{2}}^{2}+2 \omega(1-\omega) \int_{0}^{T}\|\nabla u\|_{L^{2}}^{2}+2 \omega(1-\omega) \int_{0}^{T} \int_{\mathbb{R}^{2}} \mu \\
&+\frac{W e}{2}\|\tau\|_{L^{2}}^{2}+\frac{W e}{4} \int_{\mathbb{R}^{2}}(\alpha+4 \beta)+\int_{0}^{T}\|\tau\|_{L^{2}}^{2}+\frac{1}{2} \int_{0}^{T} \int_{\mathbb{R}^{2}}(\alpha+4 \beta) \\
&=\omega\left\|u_{0}\right\|_{L^{2}}^{2}+\frac{W e}{2}\left\|\tau_{0}\right\|_{L^{2}}^{2}+\frac{W e}{4} \int_{\mathbb{R}^{2}}\left(\alpha_{0}+4 \beta_{0}\right) .
\end{aligned}
$$

If the latter holds uniformly with respect to $W e$, then we get uniform bounds on $\mu, \alpha$ and $\beta$ in $L^{1}\left((0, \infty) ; L^{1}\right)$, and by $\mathrm{A} .17$

$$
|\eta| \leq \frac{1}{2}(\mu+\alpha), \quad|\delta| \leq \frac{1}{2}(\mu+\alpha)
$$

we bound $\eta$ and $\delta$ uniformly in $W e$ in $L^{1}\left((0, \infty) ; L^{1}\right)$. The same holds of course for $\lambda$ and $\varepsilon$ as well. In order to show A.21, we need to prove that $\mu, \alpha$ and $\beta$ belong to $L_{l o c}^{1}\left((0, \infty) ; L^{1}\right)$. According to the results above,

$$
\alpha, \beta \in L^{\infty}\left((0, \infty), L^{\frac{q}{2}}\right) \quad \text { and } \quad \mu \in L_{l o c}^{2}\left((0, \infty), L^{\frac{q}{2}}\right) \quad(\text { see } \mathrm{A} .18) .
$$

However, these bounds are not uniform in $W e$, and do not imply a $L_{l o c}^{1}\left((0, \infty) ; L^{1}\right)$ bound. Here a stronger result is needed on the sequence $\tau_{n}$ : 
Result C (P.-L. Lions and N. Masmoudi). We assume (A.14), i.e. the uniform equiintegrability of $\tau_{0, n}$ in $L^{2}$. Then, $\tau_{n}$ is uniformly equiintegrable in $L_{l o c}^{\infty}\left((0, \infty) ; L^{2}\right)$, i.e. for all $T>0$,

$$
\sup _{t \in(0, T)} \sup _{n} \int_{\left|\tau_{n}\right| \geq M}\left|\tau_{n}\right|^{2} \stackrel{M \rightarrow \infty}{\longrightarrow} 0 \text {. }
$$

We refer to [24] Section III.3 for details concerning the proof. Let us point out the main idea. It is to consider $\tau_{n}$ as a solution of the linear system

$$
\left\{\begin{aligned}
\partial_{t} \tau_{n}+u \cdot \nabla \tau_{n}+\tau_{n} W(u)-W(u) \tau_{n}+\frac{1}{W e} \tau_{n} & =\frac{2 \omega D(u)}{W e} \\
\tau_{n}(0, \cdot) & =\tau_{0, n}
\end{aligned}\right.
$$

with $u$ fixed, which yields an affine mapping $K_{u}: \tau_{0, n} \longmapsto \tau_{n}$ depending on $u$. Yet, $K_{u}$ satisfies estimates independent of $u$. For $R$ an auxilliary parameter, one then decomposes the initial data into

$$
\tau_{0, n}=\tau_{0, n} 1_{\left|\tau_{0, n}\right|<R}+\tau_{0, n} 1_{\left|\tau_{0, n}\right| \geq R}
$$

and bounds

$$
\int_{\left|\tau_{n}\right| \geq M}\left|\tau_{n}\right|^{2} \leq \int_{\left|\tau_{n}\right| \geq M}\left|K_{u}\left(\tau_{0, n} 1_{\left|\tau_{0, n}\right|<R}\right)\right|^{2}+\int_{\mathbb{R}^{2}}\left|K_{u}\left(\tau_{0, n} 1_{\left|\tau_{0, n}\right| \geq R}\right)\right|^{2} .
$$

The second integral is made small for $R$ large thanks to (A.14). The first is small in the limit $M \rightarrow \infty$. Note that up to this point, we do not take care on the dependence on $W e$.

We deduce from A.22, that

$$
\begin{array}{cc}
a_{n} \text { curl }_{n}-\text { acurlu, } & b_{n} \text { curl }_{n}-\text { bcurlu } \\
\left(\partial_{1} u_{1, n}-\partial_{2} u_{2, n}\right) a_{n}-\left(\partial_{1} u_{1}-\partial_{2} u_{2}\right) a, & \left(\partial_{1} u_{2, n}+\partial_{2} u_{1, n}\right) b_{n}-\left(\partial_{1} u_{2}+\partial_{2} u_{1}\right) b
\end{array}
$$

are uniformly equiintegrable in $L_{l o c}^{1}\left((0, \infty) ; L^{1}\right)$. Therefore, they converge weakly in $L_{l o c}^{1}\left((0, \infty) ; L^{1}\right)$, and their weak limits $\delta, \varepsilon, \eta$ and $\lambda$ belong to $L_{l o c}^{1}\left((0, \infty) ; L^{1}\right)$. The defect measure $\mu$ is in $L_{\text {loc }}^{1}\left((0, \infty) ; L^{1}\right)$ because of the equality A.18.

Final step. We proceed exactly as in the Section 2.1 from A.20 we get the existence of

$$
u^{0} \in L^{\infty}\left((0, \infty) ; L^{2, \sigma}\right) \cap L^{2}\left((0, \infty) ; \dot{H}^{1}\right) \quad \text { and } \quad \tau^{0} \in L^{2}\left((0, \infty) ; L^{2}\right),
$$

such that convergences analogous to (2.1) hold. Passing to the limit $W e \rightarrow 0$ in (A.19) leads to the fact that $u^{0}$ satisfies the Navier-Stokes system $(1.6)$ in the weak sense.

REMARK 10. In order to pass to the limit in the energy equality A.21, we assume moreover that $\sqrt{W e} \tau_{0}$ tends to zero in $L^{2}$ and that $W e\left(\alpha_{0}+4 \beta_{0}\right)$ tends to zero in $L^{1}$. Thus passing to the limit, using the sign of the defect measures and the obtained weak convergences, we recover the usual energy estimate for the Navier-Stokes system.

\section{REFERENCES}

[1] J. W. Barrett and S. Boyaval, Existence and approximation of a (regularized) Oldroyd-B model, Math. Models Methods Appl. Sci., 21 (2011), pp. 1783-1837.

[2] F. Berthelin And A. Vasseur, From kinetic equations to multidimensional isentropic gas dynamics before shocks, SIAM J. Math. Anal., 36 (2005), pp. 1807-1835.

[3] Y. Brenier, Convergence of the Vlasov-Poisson system to the incompressible Euler equations, Comm. Partial Differential Equations, 25 (2000), pp. 737-754.

[4] Y. Brenier, R. Natalini, And M. Puel, On a relaxation approximation of the incompressible Navier-Stokes equations, Proc. Amer. Math. Soc., 132 (2004), pp. 1021-1028 (electronic).

[5] P. Constantin and C. Foias, Navier-Stokes equations, Chicago Lectures in Mathematics, University of Chicago Press, Chicago, IL, 1988.

[6] P. Constantin and W. Sun, Remarks on Oldroyd-B and related complex fluid models, Commun. Math. Sci., $10(2012)$

[7] C. M. Dafermos, The second law of thermodynamics and stability, Arch. Rational Mech. Anal., 70 (1979), pp. $167-179$. 
[8] - Stability of motions of thermoelastic fluids, J. Thermal Stresses, 2 (1979), pp. 127-134.

[9] S. Demoulini, D. M. A. Stuart, and A. E. Tzavaras, Weak-strong uniqueness of dissipative measurevalued solutions for polyconvex elastodynamics, Arch. Ration. Mech. Anal., 205 (2012), pp. 927-961.

[10] R. J. DiPERna, Uniqueness of solutions to hyperbolic conservation laws, Indiana Univ. Math. J., 28 (1979), pp. $137-188$.

[11] Q. Du, C. LiU, AND P. Yu, FENE dumbbell model and its several linear and nonlinear closure approximations, Multiscale Model. Simul., 4 (2005), pp. 709-731 (electronic).

[12] - From micro to macro dynamics via a new closure approximation to the FENE model of polymeric fluids, Multiscale Model. Simul., 3 (2005), pp. 895-917 (electronic).

[13] M. Giga, Y. Giga, And H. Sohr, L ${ }^{p}$ estimates for the Stokes system, in Functional analysis and related topics, 1991 (Kyoto), vol. 1540 of Lecture Notes in Math., Springer, Berlin, 1993, pp. 55-67.

[14] Y. Giga And H. Sohr, Abstract $L^{p}$ estimates for the Cauchy problem with applications to the Navier-Stokes equations in exterior domains, J. Funct. Anal., 102 (1991), pp. 72-94.

[15] F. Golse and L. Saint-Raymond, The Navier-Stokes limit of the Boltzmann equation for bounded collision kernels, Invent. Math., 155 (2004), pp. 81-161.

[16] D. Han-Kwan, Quasineutral limit of the Vlasov-Poisson system with massless electrons, Comm. Partial Differential Equations, 36 (2011), pp. 1385-1425.

[17] D. Hu And T. Lelièvre, New entropy estimates for Oldroyd-B and related models, Commun. Math. Sci., 5 (2007), pp. 909-916.

[18] B. Jourdain, C. Le Bris, T. Lelièvre, And F. Otto, Long-time asymptotics of a multiscale model for polymeric fluid flows, Arch. Ration. Mech. Anal., 181 (2006), pp. 97-148.

[19] R. Keunings, On the Peterlin approximation for finitely estensible dumbells, J. Non-Newtonian Fluid Mech., 68 (1997), pp. 85-100.

[20] C. Lattanzio And A. E. Tzavaras, Structural properties of stress relaxation and convergence from viscoelasticity to polyconvex elastodynamics, Arch. Ration. Mech. Anal., 180 (2006), pp. 449-492.

[21] — Relative entropy in diffusive relaxation, SIAM J. Math. Anal., 45 (2013), pp. 1563-1584.

[22] C. Le Bris and T. Lelièvre, Multiscale modelling of complex fluids: a mathematical initiation, in Multiscale modeling and simulation in science, vol. 66 of Lect. Notes Comput. Sci. Eng., Springer, Berlin, 2009, pp. 49-137.

[23] N. LEgER AND A. VAsseur, Relative entropy and the stability of shocks and contact discontinuities for systems of conservation laws with non-BV perturbations, Arch. Ration. Mech. Anal., 201 (2011), pp. 271-302.

[24] P. L. Lions and N. Masmoudi, Global solutions for some Oldroyd models of non-Newtonian flows, Chinese Ann. Math. Ser. B, 21 (2000), pp. 131-146.

[25] P.-L. Lions And N. Masmoudi, From the Boltzmann equations to the equations of incompressible fluid mechanics. I, II, Arch. Ration. Mech. Anal., 158 (2001), pp. 173-193, 195-211.

[26] N. Masmoudi, Global existence of weak solutions to macroscopic models of polymeric flows, J. Math. Pures Appl. (9), 96 (2011), pp. 502-520.

[27] L. Molinet and R. TAlmouk, Newtonian limit for weakly viscoelastic fluid flows of Oldroyd type, SIAM J. Math. Anal., 39 (2008), pp. 1577-1594.

[28] R. Natalini and F. Rousset, Convergence of a singular Euler-Poisson approximation of the incompressible Navier-Stokes equations, Proc. Amer. Math. Soc., 134 (2006), pp. 2251-2258.

[29] P. Oswald, Rhéophysique, ou comment coule la matière, Collection Échelles, Belin, 2005.

[30] H. C. Ö TTINGER, Beyond Equilibrium Thermodynamics, Wiley, 2005.

[31] F. Отto and A. E. Tzavaras, Continuity of velocity gradients in suspensions of rod-like molecules, Comm. Math. Phys., 277 (2008), pp. 729-758.

[32] M. Renardy, Mathematical analysis of viscoelastic flows, vol. 73 of CBMS-NSF Regional Conference Series in Applied Mathematics, Society for Industrial and Applied Mathematics (SIAM), Philadelphia, PA, 2000.

[33] J.-C. SAUT, Some remarks on the limit of viscoelastic fluids as the relaxation time tends to zero, in Trends in applications of pure mathematics to mechanics (Bad Honnef, 1985), vol. 249 of Lecture Notes in Phys., Springer, Berlin, 1986, pp. 364-369.

[34] Lectures on the mathematical theory of viscoelastic fluids. Chinese-French Summer Research Institute Project on Stress Tensor Effects on Fluid Mechanics, February 2012.

[35] J. Simon, Équations de Navier-Stokes. Cours de DEA, Université de Clermont-Ferrand, http://math.unice. $\mathrm{fr} / \sim \mathrm{j}$ simon/ 2003.

[36] R. Tемам, On the Euler equations of incompressible perfect fluids, J. Functional Analysis, 20 (1975).

[37] A. E. Tzavaras, Relative entropy in hyperbolic relaxation, Commun. Math. Sci., 3 (2005), pp. $119-132$.

[38] P. Wapperom and M. A. Hulsen, Thermodynamics of viscoelastic fluids, J. Rheol., 42 (1998), pp. 999-1019.

[39] L. E. Wedgewood And Bird R. B., From molecular models to the solution of flow problems, Ind. Eng. Chem. Res., 27 (1988), pp. 1313-1320.

[40] H.-T. YAu, Relative entropy and hydrodynamics of Ginzburg-Landau models, Lett. Math. Phys., 22 (1991), pp. $63-80$. 